Preprints of the

Max Planck Institute for

Research on Collective Goods

Bonn 2009/28

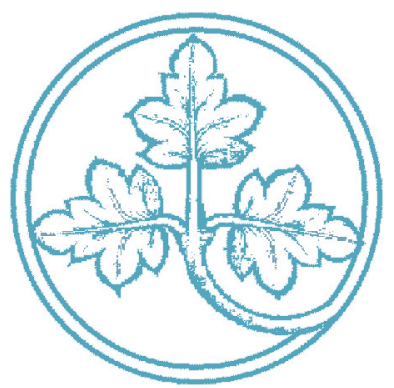

Informational Smallness and the Scope for Limiting Information Rents

Alia Gizatulina

Martin Hellwig

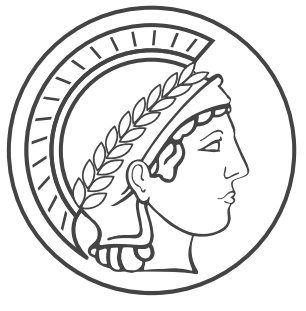




\section{Informational Smallness and the Scope for Limiting Information Rents}

Alia Gizatulina / Martin Hellwig

August 2009 


\title{
Informational Smallness and the Scope for Limiting Information Rents ${ }^{1}$
}

\author{
Alia Gizatulina ${ }^{2}$ and Martin Hellwig ${ }^{3}$ \\ Max Planck Institute for Research on Collective Goods \\ Kurt-Schumacher-Str. 10 \\ D - 53113 Bonn, Germany \\ tel: $-49-228-9141620$ \\ fax: $-49-228-9141621$
}

August 22, 2009

\footnotetext{
${ }^{1}$ Without implicating them, we thank Felix Bierbrauer, Christoph Engel, Bruno Jullien, Stephen Morris and Michael Vogt for helpful discussions and comments. 22 gizatulina@coll.mpg.de

${ }^{3}$ hellwig@coll.mpg.de
} 


\begin{abstract}
For an incomplete-information model of public-good provision with interim participation constraints, we show that efficient outcomes can be approximated, with approximately full surplus extraction, when there are many agents and each agent is informationally small. The result holds even if agents' payoffs cannot be unambiguously inferred from their beliefs, i.e., even if the so-called BDP property ("Beliefs Determine Preferences") of Neeman (2004) does not hold. The contrary result of Neeman (2004) rests on an implicit uniformity requirement that is incompatible with the notion that agents are informationally small because there are many other agents who have information about them.
\end{abstract}

JEL Classification: D40, D44, D80, D82

Keywords: surplus extraction, mechanism design, BDP, informational smallness, correlated information. 


\section{Introduction}

A central theme of the economics of information concerns the ability of agents to earn rents because they have private information that cannot be exploited unless they are given an information rent. Thus, a seller cannot appropriate the entire surplus from a sale if the potential buyers have independent private values.

However, Crémer and McLean (1988) have shown that, in models with correlated private values, information rents can be made to disappear so that, if there are two or more potential buyers, the seller can extract all surplus. Crémer and McLean (1988) assumed finite type sets, but McAfee and Reny (1992) have extended their result to models with a continuum of types.

The analysis of Crémer and McLean (1988) has been challenged by Neeman (2004) and Heifetz and Neeman (2006). They show that the conclusions of Crémer and McLean (1988) depend on a peculiar property of their specification of information. Under this property, which they summarize by the phrase "beliefs determine preferences" (BDP), any agent's payoff can be precisely inferred from his beliefs about the rest of the world. Thus, if there are two states of the world in which an agent has different payoffs, then, in these two states, he must also have different beliefs about the rest of the world. Heifetz and Neeman (2006) show that, without this property, full surplus extraction is impossible. They also suggest that failures of BDP are generic.

Neeman (2004) goes one step further and argues that, with certain failures of BDP, it may not even be possible to extract any significant surplus at all. Thus, for a model of voluntary public-good provision with interim participation constraints, he shows that, for certain failures of BDP, feasible and incentive-compatible public-good provision levels are close to zero when there are many agents. His version of public-good provision with correlated private values thus behaves like the independent-private-values model of Mailath and Postlewaite (1990).

We want to take issue with this claim. In a world with correlated types, some information about a given agent's payoff can be obtained from the 
messages that are sent by other agents. This information may be used to discipline the agent and to limit his information rent. In particular, therefore, an agent's ability to extract information rents is small if the agent is informationally small, i.e. if the agent's information does not add much to the information that can be obtained from other agents. This is likely to be the case when there are many agents and the aggregate information of all agents but one provides a fairly precise estimate of the remaining agent's information. Neeman (2004) neglects the possibility that such additional information may reduce the individual agents' ability to extract information rents.

To substantiate our criticism, we study a version of the public-good provision problem in which all agents have information about all other agents, and we show that the information effects of having more participants outweigh the free-rider effects that drive the results of Mailath and Postlewaite (1990) or Neeman (2004). If the number of participants is sufficiently large, it is possible to implement an approximately efficient, individually rational allocation with a positive level of public-good provision.

Our criticism should not be read as saying that Neeman's theorem is invalid. His theorem is valid, but his specification of failures of BDP involves an implicit additional assumption that we find problematic. In Neeman's analysis, BDP fails in such a way that, for each agent and each state of the world in which the agent gets a positive payoff from the public good, there is another state of the world in which the agent has the same beliefs, but the payoff he draws from the public good is zero. Indeed, he assumes that the conditional probability of the zero-payoff state given the agent's beliefs is bounded away from zero, regardless of what the agent's beliefs might be and regardless of how many other agents there are in the economy.

The assumption that failures of BDP are independent of the number of participants is problematic. This requirement excludes models in which each agent has a vector of noisy signals about the other agents so that, if the number of agents is large, the aggregate of all agents' noisy signals provides very precise information about any one agent's payoff parameters. In such models, the agent's beliefs about the average of the other agents' noisy signals about his own payoff parameter reflect the value of the payoff 
parameter itself. If the agent also has information about the noise in the other agents' signals, BDP may still fail because a given set of beliefs may be compatible with multiple combinations of payoffs parameters and additional information. However, the extent of the failure depends on how many other agents there are: If there are many of them and the noise in their signals is independent, the association of the agent's beliefs about the average of the other agents' noisy signals about his own payoff parameter with the value of the payoff parameter itself will be very close, and the conditional probabilities of his payoff parameter given his beliefs will be close to degenerate. Failures of BDP are less and less important when the number of participants becomes large.

Our analysis is inspired by the literature on informational smallness. ${ }^{1}$ Heuristically, an agent is informationally small if, even without the agent's private information, it is possible to implement at least approximately efficient outcomes. In the literature, this concept of informational smallness is related to the role played by the individual agent's private information in determining aggregate outcomes. Thus, in a model of public-good provision, an agent is informationally small in this sense if his influence on the decision to provide the public good or not is small, e.g., because his information has not much of an effect on the aggregate valuation for the public good. In our analysis, by contrast, informational smallness also concerns the role played by the individual agent's private information in determining the amount that he can be made to contribute to the public good. Without informational smallness, information rents might be such that, under the given participation constraints, it would be impossible to raise any significant funds for public-good provision at all.

The mechanisms that we use to implement approximately efficient, individually rational allocations are different from the mechanisms used by Crémer and McLean (1988) or, for that matter, McLean and Postlewaite (2002). In Crémer and McLean (1988), differences in beliefs induce differences in attitudes towards bets or, more generally, state-contingent payment schemes. These differences in attitudes towards state-contingent payment

\footnotetext{
${ }^{1}$ Palfrey and Srivastava (1986), Gul and Postlewaite (1992), McLean and Postlewaite (2002).
} 
schemes are used to alleviate incentive constraints. If differences in payoff parameters are aligned with differences in beliefs, it is possible to extract rents, at least in expected-value terms, by providing agents with type-dependent state-contingent payment schemes that are designed in such a way that any one type's expected payment reflects this type's willingness to pay and incentive compatibility is ensured by tailoring the state-contingencies of payments to the different beliefs of the different types. ${ }^{2}$

By contrast, we rely on scoring rules that directly penalize agents if their reports about their own payoffs differ too widely from the average reports of other agents about their payoffs. As discussed by Miller, Pratt, Zeckhauser, and Johnson (2007) such scoring rules induce agents to be approximately honest in reporting their payoffs if the weights given to the scoring components in payment rules are large. In principle, such large weights given to scoring components in payment rules are inefficient because they induce agents to suffer penalties if the other agents' information about them is noisy. However, when there are many other agents and the noise terms in their signals are conditionally independent, the law of large numbers implies that an average of these signals involves little noise. In this case, the efficiency loss from having a large weight given to scoring components in payment rules will be negligible.

Our analysis should not be interpreted as saying that informational smallness is of practical importance in dealing with problems of private information. Our sole objective is to clarify the relation between the different concepts, informational smallness and failures of BDP, and their implications for the ability of agents to obtain information rents.

In the following, Section 2 lays out the basic framework of our analysis, the underlying economic model, as well as the specification of information and beliefs. Section 3 provides our main result, showing that first-best implementation can be approximated if the economy is large and each agent is informationally small. In the concluding remarks in Section 4, we consider

\footnotetext{
${ }^{2}$ The BDP property is needed for such a scheme to eliminate all information rents: If there are two states of the world in an agent has different payoffs and the same beliefs about the rest of the world, there is no way to prevent the agent from earning an information rent corresponding to the difference between his payoffs in these two states of the world.
} 
the relation between our result and the literature on informational smallness. In this section, we also return to the question of what precisely is the relation between informational smallness and BDP.

\section{The Basic Framework}

\subsection{The Underlying Economic Model}

We consider a model with one private good and one public good. The public good comes as a single indivisible unit. Installing it involves a per capita cost equal to $K>0$ units of the private good. There are $n$ agents $i=1, . ., n$. Agent $i$ has a quasi-linear utility function

$$
U_{i}\left(Q, \theta_{i}, m_{i}\right)=\theta_{i} Q+m_{i}
$$

where $Q \in\{0,1\}$ is the level of public-good provision, $m_{i}$ is the amount of private-good consumption, and $\theta_{i}$ is a payoff parameter. Feasibility considerations impose the constraint

$$
n K Q+\sum_{i=1}^{n} m_{i} \leq n Y,
$$

where $n Y$ is an exogenously given measure of aggregate resource availability. If the allocation $\left(Q, m_{1}, \ldots m_{I}\right)$ is to be ex post Pareto efficient, one must therefore have

$$
Q=1 \text { if } \sum_{i=1}^{n} \theta_{i}>n K,
$$

and

$$
Q=0 \text { if } \sum_{i=1}^{n} \theta_{i}<n K,
$$

To implement an allocation that is ex post efficient, one needs to know $\sum_{i=1}^{n} \theta_{i}$. However, for each $i$, the preference parameter $\theta_{i}$ is private information of agent $i$. The question is to what extent this information can be extracted and used to determine public-good provision. 


\subsection{Information and Beliefs}

To model information, we assume that there is some underlying space $\Omega$ of possible states of the world, and that all beliefs are derived from a common prior $F$ on this space. The payoff parameter $\theta_{i}$ of agent $i$ is the realization of a random variable $\tilde{\theta}_{i}$ on $\Omega$. The agent also observes two sets of signals, first-order signals $s_{j}^{i}, j \neq i$, and second-order signals $\sigma_{j}^{i}, j \neq i$, with the interpretation that the first-order signals contain information about the other agents' payoff parameters, and the second-order signals contain information about the other agents' first-order signals about his own payoff parameter. The $s_{j}^{i}, \sigma_{j}^{i}$ are the realizations of random variables $\tilde{s}_{j}^{i}, \tilde{\sigma}_{j}^{i}$ such that, for $j \neq i$

$$
\tilde{s}_{j}^{i}=\tilde{\theta}_{j}+\tilde{\varepsilon}_{j}^{i}
$$

and

$$
\tilde{\sigma}_{j}^{i}=\tilde{s}_{i}^{j}+\tilde{\eta}_{j}^{i}
$$

We assume that the random variables $\tilde{\theta}_{1}, \ldots, \tilde{\theta}_{n}$ take values in a compact subset $\Theta \subset \mathbb{R}$. They are independent and identically distributed, and the mean $E \tilde{\theta}_{i}$ exceeds the per-capita public-good provision cost $K$. We also assume that the random variables $\tilde{s}_{j}^{i}, i=1, \ldots, n, j \neq i$, take values in a compact set $S \subset \mathbb{R}$. Conditional on $\tilde{\theta}_{1}, \ldots, \tilde{\theta}_{n}$, the random variables $\tilde{\varepsilon}_{j}^{i}$ and $\tilde{\eta}_{j}^{i}, i=1, \ldots, n, j \neq i$, are independent, with means $E\left[\tilde{\varepsilon}_{j}^{i} \mid \tilde{\theta}_{1}, \ldots, \tilde{\theta}_{n}\right]=0$ and $E\left[\tilde{\eta}_{j}^{i} \mid \tilde{\theta}_{1}, \ldots, \tilde{\theta}_{n}\right]=0$. Thus, $E\left[\tilde{s}_{j}^{i} \mid \tilde{\theta}_{i}\right]=\tilde{\theta}_{i}$ and $E\left[\tilde{\sigma}_{j}^{i} \mid \tilde{s}_{i}^{j}\right]=\tilde{s}_{i}^{j}$ for all $i$ and all $j \neq i$. $^{3}$

In this setting, one can think of the type of agent $i$ as a vector

$$
t_{i}=\left(\theta_{i}, s_{1}^{i}, \ldots s_{n}^{i}, \sigma_{1}^{i}, \ldots, \sigma_{n}^{i}\right)
$$

of payoff parameters and signal observations. Given the information that the random variables $\tilde{\theta}_{i}, \tilde{s}_{1}^{i}, \ldots, \tilde{s}_{n}^{i}, \tilde{\sigma}_{1}^{i}, \ldots, \tilde{\sigma}_{n}^{i}$ take the values $\theta_{i}, s_{1}^{i}, \ldots s_{n}^{i}, \sigma_{1}^{i}, \ldots, \sigma_{n}^{i}$, the agent updates his expectations, replacing the prior $F$ by a regular conditional distribution on $\Omega$ given this information. Denote this conditional

\footnotetext{
${ }^{3}$ We might actually replace (5) and (6) by the assumption that $E\left[\tilde{s}_{j}^{i} \mid \tilde{\theta}_{i}\right]=\tilde{\theta}_{i}$ and $E\left[\tilde{\sigma}_{j}^{i} \mid \tilde{s}_{i}^{j}\right]=\tilde{s}_{i}^{j}$ for all $i$ and all $j \neq i$ and that the disturbance terms $\tilde{s}_{j}^{i}-E\left[\tilde{s}_{j}^{i} \mid \tilde{\theta}_{i}\right], j \neq i$, are independent.
} 
distribution as $B_{i}\left(t_{i}\right)$. The agent's conditional beliefs about the other agents are then given by the joint distribution of the random variables $\tilde{t}_{j}, j \neq i$, that is induced by the distribution $B_{i}\left(t_{i}\right)$. We denote this conditional joint distribution of the random variables $\tilde{t}_{j}, j \neq i$, as $b_{i}\left(t_{i}\right)$.

\subsection{The BDP Property}

As mentioned in the introduction, the analysis of Crémer and McLean (1988) rests on the observation that people's willingness to accept bets depends on their beliefs. Such bets can be used to extract the belief $b_{i}\left(t_{i}\right)$ of agent $i$. If this information can be used to infer the agent's payoff type $\theta_{i}$, there is no need to provide agent $i$ with a rent for divulging $\theta_{i}$. Neeman (2004) has referred to this feature of the Crémer and McLean (1988) model as the BDP property ("beliefs determine preferences"). In our setting, with a common prior on the underlying probability space, the BDP property is a property of the prior $F$ and the random variables $\tilde{t}_{1}, \ldots \tilde{t}_{n}$. We say that $F$ and $\tilde{t}_{1}, \ldots \tilde{t}_{n}$ exhibit the $B D P$ property if, for $i=1, \ldots, n$, one has

$$
E\left[\tilde{\theta}_{i}(\cdot) \mid b_{i}\left(\tilde{t}_{i}\right)\right]=\tilde{\theta}_{i},
$$

and

$$
\operatorname{Var}\left[\tilde{\theta}_{i}(\cdot) \mid b_{i}\left(\tilde{t}_{i}\right)\right]=E\left[\left(\tilde{\theta}_{i}-E\left[\tilde{\theta}_{i}(\cdot) \mid b_{i}\left(\tilde{t}_{i}\right)\right]\right)^{2} \mid b_{i}\left(\tilde{t}_{i}\right)\right]=0 .
$$

$F$-almost surely, so that, the distribution of $\tilde{\theta}_{i}(\cdot)$ conditional on the event $b\left(\tilde{t}_{i}\right)=b_{i}$ is degenerate and assigns all mass to a single value $\theta_{i}$; thus, $\tilde{\theta}_{i}(\omega)=\theta_{i}$ for any $\omega$ such that $b\left(\tilde{t}_{i}(\omega)\right)=b_{i}$.

In our model, BDP does not generally hold. Specifically, under our independence assumptions, we find that for each $\tilde{t}_{j}=\left(\tilde{\theta}_{j}, \tilde{s}_{1}^{j}, \ldots, \tilde{s}_{n}^{j}\right)$ :

- Agent $i$ 's conditional beliefs about $\tilde{\theta}_{j}$ are determined by $\tilde{s}_{j}^{i}$; they have nothing to do with $\tilde{\theta}_{i}$.

- Agent $i$ 's conditional beliefs about $\tilde{s}_{i}^{j}$ are determined by $\tilde{\theta}_{i}$ and $\tilde{\sigma}_{j}^{i}$; it is not generally possible to disentangle the influence of $\tilde{\theta}_{i}$ and $\tilde{\sigma}_{j}^{i}$ and to infer $\tilde{\theta}_{i}$ from the agent's beliefs about $\tilde{s}_{i}^{j}$.

- Agent $i$ 's conditional beliefs about $\tilde{s}_{k}^{j}$, for $k \neq i$, are equal to his prior beliefs; they have nothing to do with $\tilde{\theta}_{i}$. 
- Agent $i$ 's conditional beliefs about $\tilde{\sigma}_{k}^{j}$, for any $k$, are equal to his prior beliefs; in particular, they have nothing to do with $\tilde{\theta}_{i}$.

\section{An Implementation Theorem}

Because $\tilde{\theta}_{1}, \ldots, \tilde{\theta}_{n}$ are independent and identically distributed, with $E \tilde{\theta}_{i}>K$, we know that, if $n$ is large, then, with a probability close to one, it is efficient for the public to be provided. In the following, we show that this efficient outcome can be approximately achieved if $n$ is large. Moreover, under the mechanism we consider, each agent's interim expectation of the benefits from participating is strictly positive. These findings will stand in contrast to the result in Neeman (2004), where, in the absence of BDP, participation constraints preclude a positive level of public-good provision.

We consider direct mechanisms that do not induce truthtelling but only $\varepsilon$-truthtelling. We consider a class of such mechanisms parametrized by two parameters $\delta>0$ and $w>0$, as well as the number of participants $n$. Each participant $i$ is asked to submit a report

$$
\hat{t}_{i}=\left(\hat{\theta}_{i}, \hat{s}_{1}^{i}, \ldots \hat{s}_{n}^{i}, \hat{\sigma}_{1}^{i}, \ldots, \hat{\sigma}_{n}^{i}\right)
$$

about his type. Given the reports $\hat{t}_{1}, \ldots, \hat{t}_{n}$, the probability of public-good provision is fixed at

$$
q\left(\hat{t}_{1}, \ldots, \hat{t}_{n} \mid \delta, w, n\right)=g\left(\frac{1}{\delta}\left(\frac{1}{n} \sum_{k} \hat{\theta}_{k}-K\right)\right)
$$

where $g(\cdot)$ is a twice continuously differentiable nondecreasing function satisfying

$$
g(x)=0 \text { if } x \leq 0 \text { and } g(x)=1 \text { if } x \geq 1 .
$$

Payments are conditioned on whether the public good is provided or not. If the public good is not provided, agent $i$ pays

$p_{i}^{0}\left(\hat{t}_{1}, \ldots, \hat{t}_{n} \mid \delta, w, n\right)=\frac{w}{2}\left(\hat{\theta}_{i}-\frac{1}{n-2} \sum_{j \neq i, i+1} \hat{s}_{i}^{j}\right)^{2}-\frac{w}{2}\left(\hat{\theta}_{i-1}-\frac{1}{n-2} \sum_{j \neq i-1, i} \hat{s}_{i-1}^{j}\right)^{2}$, 
where, as usual, the indices $i-1$ and $i+1$ are to be understood modulo $n$, with $n+1:=1$, and $1-1:=n$. If the public good is provided, the agent pays

$$
p_{i}^{1}\left(\hat{t}_{1}, \ldots, \hat{t}_{n} \mid \delta, w, n\right)=p_{i}^{0}\left(\hat{t}_{1}, \ldots, \hat{t}_{n} \mid \delta, w, n\right)+\hat{\theta}_{i}-\left(\frac{1}{n} \sum_{j=1}^{n} \hat{\theta}_{j}-K\right) .
$$

Before we proceed with the analysis, we make a few comments to explain the mechanism.

- The mechanism makes public-good provision depend only on the reports $\hat{\theta}_{i}$ that agents send about their own payoff parameters. From (10) and (11), one infers that

$$
q\left(\hat{t}_{1}, \ldots, \hat{t}_{n} \mid \delta, w, n\right)=0 \text { if } \frac{1}{n} \sum_{k} \hat{\theta}_{k} \leq K
$$

and

$$
q\left(\hat{t}_{1}, \ldots, \hat{t}_{n} \mid \delta, w, n\right)=1 \text { if } \frac{1}{n} \sum_{k} \hat{\theta}_{k} \geq K+\delta .
$$

The public good is provided for sure if the average of the reports $\hat{\theta}_{1}, \ldots, \hat{\theta}_{n}$ exceeds $K+\delta$; it is for sure not provided if the average of these reports is less than $K$. Upon comparing (14) and (15) with (3) and (4), one sees that the provision rule (10) comes close to implementing first-best if $\delta$ is close to zero and if the participants' reports about their payoffs are close to their actual payoffs. The function $g(\cdot)$ is introduced to ensure that the public-good provision rule and the payment functions are twice continuously differentiable.

- Each payment function has a public-good component and a scoring component. The parameter $w$ indicates the weight of the scoring component. Each of the two components of the payment function has a targeted part and a budget-balancing part.

- The targeted part of the public-good component requires agent $i$ to pay $\hat{\theta}_{i}$, his reported benefit from the public good, if the public good is provided. The budget-balancing part provides the agent with a share 
$\frac{1}{n}$ of the surplus $\sum_{j=1}^{n} \hat{\theta}_{j}-n K$ of the sum of targeted payments over provision costs if the public good is provided. If the public good is not provided, the public-good component of the payment function is zero.

- The targeted part of the scoring component of the payment function requires agent $i$ to pay $\frac{w}{2}$ times $\left(\hat{\theta}_{i}-\frac{1}{n-2} \sum_{j \neq i, i+1} \hat{s}_{i}^{j}\right)^{2}$, the squared deviation of his report $\hat{\theta}_{i}$ about his payoff parameter from the crosssection average of the reports of agents other than $i$ and $i+1$ about the signals they have received about his payoff parameter. The budgetbalancing part makes agent $i$ the recipient of the targeted part of the scoring component of agent $i-1$ 's payment.

- The mechanism makes no use of the messages $\hat{\sigma}_{j}^{i}$ that agents send about the signals they have received about the signals that other agents have about them. Efficiency could presumably be improved if this information was also used.

Turning to the actual analysis, we first note that, for any $\delta, w$, and $n$, regardless of what the reports $\hat{t}_{1}, \ldots, \hat{t}_{n}$ may be, the mechanism that is determined by $\delta, w$, and $n$ has a balanced budget.

Lemma 3.1 For any $\delta, w$, and $n$, the payment functions $p_{i}^{0}(\cdot \mid \delta, w, n), p_{i}^{1}(\cdot \mid \delta, w, n)$, $i=1, \ldots, n$, satisfy

$$
\sum_{i} p_{i}^{0}\left(\hat{t}_{1}, \ldots, \hat{t}_{n} \mid \delta, w, n\right)=0
$$

and

$$
\sum_{i} p_{i}^{1}\left(\hat{t}_{1}, \ldots, \hat{t}_{n} \mid \delta, w, n\right)=n K
$$

for all $\hat{t}_{1}, \ldots, \hat{t}_{n}$.

Proof. (16) follows immediately from (12). From (13) and (16), we also have

$$
\sum_{i} p_{i}^{1}\left(\hat{t}_{1}, \ldots, \hat{t}_{n} \mid \delta, w, n\right)=\sum_{i} \hat{\theta}_{i}-\sum_{j} \hat{\theta}_{j}+n K=n K
$$


Turning to agents' payoffs, we assume that each agent has an initial endowment $Y$. If a mechanism for public-good provision requires the agent to pay the amount $p_{i}$, his private-good consumption is reduced from $Y$ to $m_{i}=Y-p_{i}$. His utility is then $\theta_{i} Q+Y-p_{i}$, his net payoff relative to the situation without a mechanism for public-good provision is $\theta_{i} Q-p_{i}$. Thus, under the mechanisms that we are considering, agent $i$ 's net payoff function is given by the equation

$$
u_{i}\left(\hat{t}_{1}, \ldots, \hat{t}_{n} \mid t_{i}, \delta, w, n\right)=q\left(\hat{t}_{1}, \ldots, \hat{t}_{n} \mid \delta, w, n\right) \theta_{i}-p_{i}\left(\hat{t}_{1}, \ldots, \hat{t}_{n} \mid \delta, w, n\right),
$$

where

$$
\begin{aligned}
p_{i}\left(\hat{t}_{1}, \ldots, \hat{t}_{n} \mid \delta, w, n\right)= & \left(1-q\left(\hat{t}_{1}, \ldots, \hat{t}_{n} \mid \delta, w, n\right)\right) p_{i}^{0}\left(\hat{t}_{1}, \ldots, \hat{t}_{n} \mid \delta, w, n\right) \\
& +q\left(\hat{t}_{1}, \ldots, \hat{t}_{n} \mid \delta, w, n\right) p_{i}^{1}\left(\hat{t}_{1}, \ldots, \hat{t}_{n} \mid \delta, w, n\right)
\end{aligned}
$$

is the agent's expected payment. Using (10) and (19), we compute

$$
\begin{aligned}
& u_{i}\left(\hat{t}_{1}, \ldots, \hat{t}_{n} \mid t_{i}, \delta, w, n\right) \\
= & g\left(\frac{1}{\delta}\left(\frac{1}{n} \sum_{j} \hat{\theta}_{j}-K\right)\right)\left(\theta_{i}-\hat{\theta}_{i}+\frac{1}{n} \sum_{j} \hat{\theta}_{j}-K\right) \\
& -\frac{w}{2}\left(\hat{\theta}_{i}-\frac{1}{n-2} \sum_{j \neq i, i+1} \hat{s}_{i}^{j}\right)^{2}+\frac{w}{2}\left(\hat{\theta}_{i-1}-\frac{1}{n-2} \sum_{j \neq i-1, i} \hat{s}_{i-1}^{j}\right)^{2}
\end{aligned}
$$

We can think of $u_{i}\left(\cdot \mid t_{i}, \delta, w, n\right)$ as agent $i$ 's payoff function in a game of imperfect information in which nature chooses the realizations $t_{1}, \ldots, t_{n}$ of the random variables $\tilde{t}_{1}, \ldots, \tilde{t}_{n}$, each agent $i$ observes his own type $t_{i}$, and then the different agents simultaneously and independently choose their reports $\hat{t}_{1}\left(t_{i}\right), \ldots, \hat{t}_{n}\left(t_{n}\right)$. A constellation $\left(\hat{t}_{1}(\cdot), \ldots, \hat{t}_{n}(\cdot)\right)$ of reporting strategies corresponds to a Bayes-Nash equilibrium of this game with parameters $\delta, w$, and $n$ if and only if, for each $i$, the strategy $\hat{t}_{i}(\cdot)$ is a best response to the strategies $\hat{t}_{j}(\cdot)$ of agents other than $i$; this requires that

$$
\begin{aligned}
& E\left[u_{i}\left(\hat{t}_{1}\left(\tilde{t}_{1}\right), \ldots, . \hat{t}_{i-1}\left(\tilde{t}_{-1}\right), \hat{t}_{i}\left(t_{i}\right), \hat{t}_{i+1}\left(\tilde{t}_{i+1}\right), \ldots, \hat{t}_{n}\left(\tilde{t}_{n}\right) \mid t_{i}, \delta, w, n\right) \mid \tilde{t}_{i}=t_{i}\right] \\
\geq & E\left[u_{i}\left(\hat{t}_{1}\left(\tilde{t}_{1}\right), \ldots \hat{t}_{i-1}\left(\tilde{t}_{-1}\right), \hat{t}_{i}, \hat{t}_{i+1}\left(\tilde{t}_{i+1}\right), \ldots, \hat{t}_{n}\left(\tilde{t}_{n}\right) \mid t_{i}, \delta, w, n\right) \mid \tilde{t}_{i}=t_{i}\right]
\end{aligned}
$$


for (almost) all $t_{i}$ and all reports $\hat{t}_{i}^{\prime}$.

Proposition 3.2 below shows that, for any $\delta$ and $n$, a Bayes-Nash equilibrium of the game with parameters $\delta, w$, and $n$ exists if $w$ is sufficiently large. Moreover, this equilibrium involves truthtelling in the reports $\hat{s}_{j}^{i}\left(t_{i}\right), \hat{\sigma}_{j}^{i}\left(t_{i}\right), j \neq$ $i$, that agent $i$ sends about the signals $s_{j}^{i}, \sigma_{j}^{i}, j \neq i$, that he has received about other agents. If $w$ and $n$ are large, the equilibrium also involves approximate truthtelling in the report $\hat{\theta}_{i}\left(t_{i}\right)$ that the agent sends about his own payoff parameter $\theta_{i}$.

Truthtelling in $\hat{s}_{j}^{i}\left(t_{i}\right), \hat{\sigma}_{j}^{i}\left(t_{i}\right), j \neq i$, is trivially obtained from the observation that the payoff $u_{i}\left(\hat{t}_{1}, \ldots, \hat{t}_{n} \mid t_{i}, \delta, w\right)$ of agent $i$ does not, in fact, depend on the messages $\hat{s}_{j}^{i}, \hat{\sigma}_{j}^{i}, j \neq i$. Approximate truthtelling in $\hat{\theta}_{i}\left(t_{i}\right)$ is obtained from the consideration that, if $w$ is sufficiently large, the report $\hat{\theta}_{i}\left(t_{i}\right)$ is largely determined by the desire to keep the scoring component of the payment $p_{i}\left(\hat{t}_{1}, \ldots, \hat{t}_{n} \mid \delta, w, n\right)$ as low as possible. For this purpose, $\hat{\theta}_{i}\left(t_{i}\right)$ is chosen to be close to the agent's expectation of the average $\frac{1}{n-2} \sum_{j \neq i, i+1} s_{i}^{j}$ of other agents' reports about the signals they have received about $\theta_{i}$. If $n$ is large, then, by the law of large numbers, this expectation is close to $\theta_{i}$. Whereas the information that the agent has from observing the additional signals $\sigma_{j}^{i}$ $j \neq i$, is important when he forms his expectations about any one of the other agents' signals $s_{i}^{j}$, this additional information is unimportant, and only $\theta_{i}$ matters when he is forming expectations about the average $\frac{1}{n-2} \sum_{j \neq i, i+1} s_{i}^{j}$, when $n$ is large.

To make these ideas precise, we need some additional notation. Recalling that the range $\Theta$ of the random variables $\tilde{\theta}_{i}, i=1, \ldots, n$, is compact, let $\bar{\theta}>0$ be such that $|\theta| \leq \bar{\theta}$ for all $\theta \in \Theta$. Similarly, let $\bar{s}>0$ be such that $|s| \leq \bar{s}$ for all $s$ belonging to the range $S$ of the random variables $\tilde{s}_{j}^{i}, i=1, \ldots, n, j \neq i$. Further, we specify $G^{1}$ and $G^{2}$ so that the derivatives of the function $g(\cdot)$ satisfy

$$
0 \leq g^{\prime}(x) \leq G^{1} \text { and }\left|g^{\prime \prime}(x)\right| \leq G^{2}
$$

for all $x \in \mathbb{R}$. By (11),

$$
G^{1}>1
$$


Proposition 3.2 Suppose that

$$
w \delta^{2}>G^{2}\left(\bar{\theta}+\bar{s}+2 \delta+\frac{G^{1}}{w}\right)+2 G^{1} \delta .
$$

Then the game with parameters $\delta, w$, and $n$ has a Bayes-Nash equilibrium $\left(\hat{t}_{1}(\cdot \mid \delta, w, n), \ldots, \hat{t}_{n}(\cdot \mid \delta, w, n)\right)$ with the following properties:

(a) For all agents $i$ and all types $t_{i}=\left(\theta_{i}, s_{1}^{i}, \ldots s_{n}^{i}, \sigma_{1}^{i}, \ldots, \sigma_{n}^{i}\right)$ of agent $i$, $\hat{s}_{j}^{i}\left(t_{i} \mid \delta, w, n\right)=s_{j}^{i}$ and $\hat{\sigma}_{j}^{i}\left(t_{i} \mid \delta, w, n\right)=\sigma_{j}^{i}$ for all $j \neq i$.

(b) For all agents $i$ and all types $t_{i}=\left(\theta_{i}, s_{1}^{i}, \ldots s_{n}^{i}, \sigma_{1}^{i}, \ldots, \sigma_{n}^{i}\right)$ of agent $i$, the report $\hat{\theta}_{i}\left(t_{i}\right)$ satisfies the equation

$$
\begin{gathered}
E\left[\left(\theta_{i}-\frac{n-1}{n} \hat{\theta}_{i}\left(t_{i}\right)+\frac{1}{n} \sum_{k \neq i} \hat{\theta}_{k}\left(\tilde{t}_{k}\right)-K\right) \frac{g^{\prime}}{n \delta} \mid \tilde{t}_{i}=t_{i}\right] \\
-\frac{n-1}{n} E\left[g \mid \tilde{t}_{i}=t_{i}\right]-w\left(\hat{\theta}_{i}\left(t_{i}\right)-\frac{1}{n-2} \sum_{j \neq i, i+1} E\left[\tilde{s}_{i}^{j} \mid \tilde{t}_{i}=t_{i}\right]\right)=0,
\end{gathered}
$$

where both $g^{\prime}$ and $g$ are evaluated at $\frac{1}{\delta}\left(\frac{1}{n}\left(\hat{\theta}_{i}\left(t_{i}\right)+\sum_{k \neq i} \hat{\theta}_{k}\left(\tilde{t}_{k}\right)\right)-K\right)$.

The proof of this proposition is somewhat involved and is given in the appendix. Because each agent's strategy is a function indicating how his report depends on his type, we need a fixed-point argument in a suitable function space. In a similar analysis, Miller, Pratt, Zeckhauser, and Johnson (2007) give such an argument. However, in their setting, strategies can be presumed to be continuous, and they can use Schauder's fixed-point theorem on a space of continuous functions. In the absence of any assumptions about conditional expectations, we cannot presume that strategies are continuous. Therefore, we work with bounded measurable functions and use Banach's fixed-point theorem for contraction mappings. The argument exploits the special structure resulting from our distinction between the payoff parameters and the different kinds of signals. As indicated by the characterization of equilibrium strategies in (25), if the weight $w$ of the scoring component is large, the major determinant of the report $\hat{\theta}_{i}\left(t_{i}\right)$ is the conditional expectation $\frac{1}{n-2} \sum_{j \neq i, i+1} E\left[\tilde{s}_{i}^{j} \mid \tilde{t}_{i}=t_{i}\right]$ that agent $i$ has concerning the cross-section mean of the reports $\tilde{s}_{i}^{j}$ which is independent of the strategies $\hat{\theta}_{j}(\cdot)$ of the 
other agents. Thus, if $w$ is large, strategic interdependence plays only a minor role in determining reports about payoff parameters.

The following theorem exploits this structure in order to give a characterization of equilibrium strategies and equilibrium outcomes when the number of participants is large.

Theorem 3.3 Let $\delta>0$ be such that

$$
E \tilde{\theta}_{i}-K>\delta
$$

Also, for any $n$, let

$$
w_{n}:=\ln n .
$$

Then, for any sufficiently large $n$, the game with parameters $\delta, w_{n}$, and $n$ has a Bayes-Nash equilibrium $\left(\hat{t}_{1}\left(\cdot \mid \delta, w_{n}, n\right), \ldots, \hat{t}_{n}\left(\cdot \mid \delta, w_{n}, n\right)\right)$ with the properties specified in Proposition 3.2. As $n$ goes out of bounds,

$$
\begin{gathered}
\hat{\theta}_{i}\left(\tilde{t}_{i} \mid \delta, w_{n}, n\right)-\tilde{\theta}_{i} \rightarrow 0, \text { almost surely, for all } i \\
q\left(\hat{t}_{1}\left(\tilde{t}_{1}\right), \ldots, \hat{t}_{n}\left(\tilde{t}_{n}\right) \mid \delta, w_{n}, n\right) \rightarrow 1, \text { almost surely, }
\end{gathered}
$$

and

$$
\begin{gathered}
E\left[u_{i}\left(\hat{t}_{1}\left(\tilde{t}_{1} \mid \delta, w_{n}, n\right), \ldots, \hat{t}_{n}\left(\tilde{t}_{n} \mid \delta, w_{n}, n\right) \mid t_{i}, \delta, w_{n}, n\right) \mid \tilde{t}_{i}\right] \rightarrow E \tilde{\theta}_{i}-K, \\
\text { almost surely, for all } i .
\end{gathered}
$$

Before we give the proof of this theorem, we briefly discuss the intuition. If the weight $w$ of the scoring components of payment rules is large, then, as discussed before, agents attune their reports about their payoff parameters above all to their expectations $\frac{1}{n-2} \sum_{j \neq i, i+1} E\left[\tilde{s}_{i}^{j} \mid \tilde{t}_{i}=t_{i}\right]$ about the crosssection means of the reports $\tilde{s}_{i}^{j}$. By the law of large numbers, for the given specification of signals $\tilde{s}_{i}^{j}$, the cross-section means $\frac{1}{n-2} \sum_{j \neq i, i+1} E\left[\tilde{s}_{i}^{j} \mid \tilde{t}_{i}=t_{i}\right]$ must converge to the true $\tilde{\theta}_{i}$ when $n$ becomes large regardless of the information contained in the signals $\sigma_{j}^{i}, j \neq i$. The specification (27) is chosen so that the weights of the scoring components of payment rules are going out of bounds and all other terms in (25) are becoming unimportant as $n$ becomes large. However, the weights of the scoring components go out of 
bounds slowly enough so that expected payments due to errors in the other agents' signals go to zero. This argument explains the convergence claim in (28). Given this convergence to truthtelling, the rest of the theorem is straightforward.

Proof. Because $\lim _{n \rightarrow \infty} w_{n}=\infty$, we have

$$
w_{n} \delta^{2}>G^{2}\left(\bar{\theta}+\bar{s}+2 \delta+\frac{G^{1}}{w_{n}}\right)+2 G^{1} \delta
$$

for any sufficiently large $n$. The first statement of the theorem then follows from Proposition 3.2.

From (25) and (5), we further obtain

$$
\begin{aligned}
\hat{\theta}_{i}\left(\tilde{t}_{i} \mid \delta, w_{n}, n\right)-\tilde{\theta}_{i}= & \frac{1}{n-2} E \sum_{j \neq i, i+1}\left[\tilde{\varepsilon}_{i}^{j} \mid \tilde{t}_{i}\right] \\
& +\frac{1}{w_{n}} E\left[\left(\tilde{\theta}_{i}-\hat{\theta}_{i}\left(\tilde{t}_{i}\right)+\frac{1}{n} \sum_{k} \hat{\theta}_{k}\left(\tilde{t}_{k} \mid \delta, w_{n}, n\right)-K\right) \frac{g^{\prime}}{n \delta} \mid \tilde{t}_{i}\right] \\
& -\frac{1}{w_{n}} \frac{n-1}{n} E\left[g \mid \tilde{t}_{i}\right],
\end{aligned}
$$

hence

$$
\begin{aligned}
\left|\hat{\theta}_{i}\left(\tilde{t}_{i} \mid \delta, w_{n}, n\right)-\tilde{\theta}_{i}\right| \leq & \left|\frac{1}{n-2} E \sum_{j \neq i, i+1}\left[\tilde{\varepsilon}_{i}^{j} \mid \tilde{t}_{i}\right]\right| \\
& +\frac{1}{w_{n}}\left|\hat{\theta}_{i}\left(\tilde{t}_{i} \mid \delta, w_{n}, n\right)-\tilde{\theta}_{i}\right| \frac{G^{1}}{n \delta} \\
& +\frac{1}{w_{n}} \delta \frac{G^{1}}{n \delta}+\frac{1}{w_{n}} .
\end{aligned}
$$

By the strong law of large numbers for strictly stationary stochastic processes (Doob (1953), p. 464 ff.), we have

$$
\frac{1}{n-2} \sum_{j \neq i, i+1} \tilde{\varepsilon}_{i}^{j} \rightarrow 0,
$$

almost surely, as $n$ becomes large. Therefore, also

$$
\left|\frac{1}{n-2} E \sum_{j \neq i, i+1}\left[\tilde{\varepsilon}_{i}^{j} \mid \tilde{t}_{i}\right]\right| \rightarrow 0,
$$


almost surely, as $n$ becomes large. Trivially, we also have

$$
\frac{1}{w_{n}} \delta \frac{G^{1}}{n \delta}+\frac{1}{w_{n}} \rightarrow 0
$$

as $n$ becomes large. (28) follows immediately.

To prove (29), we observe that

$$
\begin{aligned}
& q\left(\hat{t}_{1}\left(\tilde{t}_{1}\right), \ldots, \hat{t}_{n}\left(\tilde{t}_{n}\right) \mid \delta, w_{n}, n\right) \\
= & g\left(\frac{1}{\delta}\left[\frac{1}{n} \sum_{j=1}^{n} \hat{\theta}_{j}\left(\tilde{t}_{j} \mid \delta, w_{n}, n\right)-K\right]\right) \\
= & g\left(\frac{1}{\delta}\left[\frac{1}{n} \sum_{j=1}^{n}\left(\hat{\theta}_{j}\left(\tilde{t}_{j} \mid \delta, w_{n}, n\right)-\tilde{\theta}_{j}\right)+\frac{1}{n} \sum_{j=1}^{n} \tilde{\theta}_{j}-K\right]\right) .
\end{aligned}
$$

By (28), the first term inside the square brackets on the right-hand side of (33) goes to zero, almost surely, as $n$ becomes large. By the strong law of large numbers, the second term inside the square brackets on the right-hand side of (33) converges to $E \tilde{\theta}_{i}-K$, almost surely, as $n$ becomes large. Since $E \tilde{\theta}_{i}-K>\delta$ and, by (11), $g\left(\frac{1}{\delta} y\right)=1$ if $y \geq \delta$, it follows that the right-hand side of (33) converges to one, almost surely, as $n$ becomes large.

To prove (30), we note that, with truthtelling about the signals $\tilde{s}_{k}^{j}$, the interim payoff expectation of agent $i$ can be written as

$$
\begin{aligned}
& E\left[u_{i}\left(\hat{t}_{1}\left(\tilde{t}_{1} \mid \delta, w_{n}, n\right), \ldots, \hat{t}_{n}\left(\tilde{t}_{n} \mid \delta, w_{n}, n\right) \mid t_{i}, \delta, w_{n}, n\right) \mid \tilde{t}_{i}=t_{i}\right] \\
= & E\left[v_{i}\left(\hat{\theta}_{1}\left(\tilde{t}_{1} \mid \delta, w_{n}, n\right), \ldots, \hat{\theta}_{n}\left(\tilde{t}_{n} \mid \delta, w_{n}, n\right) \mid \tilde{t}_{i}=t_{i}\right]\right. \\
+ & \frac{w_{n}}{2} E\left[\left(\hat{\theta}_{i-1}\left(\tilde{t}_{i-1}\right)-\frac{1}{n-2} \sum_{j \neq i-1, i} \tilde{s}_{i-1}^{j}\right)^{2} \mid \tilde{t}_{i}=t_{i}\right],
\end{aligned}
$$

where, for any $\hat{\theta}_{1}, \ldots, \hat{\theta}_{n}$ and $t_{1}, \ldots, t_{n}$,

$$
\begin{aligned}
& v_{i}\left(\hat{\theta}_{1}, \ldots, \hat{\theta}_{n} \mid t_{1}, \ldots, t_{n}, \delta, w_{n}, n\right) \\
:= & g\left(\frac{1}{\delta}\left(\frac{1}{n} \sum_{j} \hat{\theta}_{j}-K\right)\right) \times\left(\theta_{i}-\frac{n-1}{n} \hat{\theta}_{i}+\frac{1}{n} \sum_{j \neq i} \hat{\theta}_{j}-K\right) \\
& -\frac{w}{2}\left(\hat{\theta}_{i}-\frac{1}{n-2} \sum_{j \neq i, i+1} s_{i}^{j}\right)^{2} .
\end{aligned}
$$


From (35), we obtain

$$
\begin{aligned}
& E\left[v_{i}\left(\hat{\theta}_{1}\left(\tilde{t}_{1} \mid \delta, w_{n}, n\right), \ldots, \hat{\theta}_{n}\left(\tilde{t}_{n} \mid \delta, w_{n}, n\right) \mid \tilde{t}_{1}, \ldots, \tilde{t}_{n}, \delta, w_{n}, n\right) \mid \tilde{t}_{i}\right] \\
= & E\left[g \times\left(\theta_{i}-\hat{\theta}_{i}\left(t_{i} \mid \delta, w_{n}, n\right)+\frac{1}{n} \sum_{j} \hat{\theta}_{j}\left(\tilde{t}_{j} \mid \delta, w_{n}, n\right)-K\right) \mid \tilde{t}_{i}\right] \\
- & \frac{w_{n}}{2} E\left[\left(\hat{\theta}_{i}\left(\tilde{t}_{i}\right)-\frac{1}{n-2} \sum_{j \neq i, i+1} \tilde{s}_{i}^{j}\right)^{2} \mid \tilde{t}_{i}=t_{i}\right],
\end{aligned}
$$

where $g$ is evaluated at $\frac{1}{\delta}\left(\frac{1}{n} \sum_{j} \hat{\theta}_{j}\left(\tilde{t}_{j} \mid \delta, w_{n}, n\right)-K\right)$.

By (28), (29), and the strong law of large numbers, we have

$\lim _{n \rightarrow \infty} E\left[g \times\left(\theta_{i}-\hat{\theta}_{i}\left(t_{i} \mid \delta, w_{n}, n\right)+\frac{1}{n} \sum_{j} \hat{\theta}_{j}\left(\tilde{t}_{j} \mid \delta, w_{n}, n\right)-K\right) \mid \tilde{t}_{i}\right]=E \tilde{\theta}_{i}-K$,

almost surely. The first term on the right-hand side of (36) thus converges to $E \tilde{\theta}_{i}-K$. Because the second term is nonpositive, it follows that

$\lim _{N \rightarrow \infty} \sup _{n \geq N} E\left[v_{i}\left(\hat{\theta}_{1}\left(\tilde{t}_{1} \mid \delta, w_{n}, n\right), \ldots, \hat{\theta}_{n}\left(\tilde{t}_{n} \mid \delta, w_{n}, n\right) \mid \tilde{t}_{1}, \ldots, \tilde{t}_{n}, \delta, w_{n}, n\right) \mid \tilde{t}_{i}\right] \leq E \tilde{\theta}_{i}-K$,

almost surely.

Because the strategy $\hat{\theta}_{i}\left(\cdot \mid \delta, w_{n}, n\right)$ is a best response to the other agents' strategies, it must also be the case that, for any $t_{i}$, agent $i$ 's expected payoff from the report $\hat{\theta}_{i}\left(t_{i} \mid \delta, w_{n}, n\right)$ is at least as large as his expected payoff from the alternative report $\hat{\theta}_{i}^{\prime}=\theta_{i}$. His expected payoff from the latter report would be

$$
\begin{gathered}
E\left[\bar{g} \times\left(\frac{1}{n} \theta_{i}+\frac{1}{n} \sum_{j \neq i} \hat{\theta}_{j}\left(\tilde{t}_{j} \mid \delta, w_{n}, n\right)-K\right) \mid \tilde{t}_{i}=t_{i}\right] \\
-\frac{w_{n}}{2} E\left[\left(\frac{1}{n-2} \sum_{j \neq i, i+1} \tilde{\varepsilon}_{i}^{j}\right)^{2} \mid \tilde{t}_{i}=t_{i}\right] \\
+\frac{w_{n}}{2} E\left[\left(\frac{1}{n-2} \sum_{j \neq i-1, i} \tilde{\varepsilon}_{i-1}^{j}\right)^{2} \mid \tilde{t}_{i}=t_{i}\right],
\end{gathered}
$$


where $\bar{g}$ refers to the value of $g$ at the point $\frac{1}{\delta}\left(\frac{1}{n} \theta_{i}+\frac{1}{n} \sum_{j \neq i} \hat{\theta}_{j}\left(\tilde{t}_{j} \mid \delta, w_{n}, n\right)-K\right)$. By the same argument as before, the first term on the right-hand side converges to $E \tilde{\theta}_{i}-K$, almost surely, as $n$ becomes large. As for the second term, the conditional-independence and symmetry assumptions that we have imposed on $\tilde{\varepsilon}_{i}^{j}, j \neq i$, implies that this term is given as

$$
\frac{w_{n}}{2} E\left[\left(\frac{1}{n-2} \sum_{j \neq i, i+1} \tilde{\varepsilon}_{i}^{j}\right)^{2} \mid \tilde{t}_{i}=t_{i}\right]=\frac{\ln n}{2(n-2)} \operatorname{Var}\left(\tilde{\varepsilon}_{i}^{j} \mid \tilde{t}_{i}=t_{i}\right),
$$

which goes to zero as $n$ becomes large. We may therefore conclude that

$\lim _{N \rightarrow \infty} \inf _{n \geq N} E\left[v_{i}\left(\hat{\theta}_{1}\left(\tilde{t}_{1} \mid \delta, w_{n}, n\right), \ldots, \hat{\theta}_{n}\left(\tilde{t}_{n} \mid \delta, w_{n}, n\right) \mid \tilde{t}_{1}, \ldots, \tilde{t}_{n}, \delta, w_{n}, n\right) \mid \tilde{t}_{i}\right] \geq E \tilde{\theta}_{i}-K$,

almost surely. Upon combining (38) and (39), we obtain

$\lim _{n \rightarrow \infty} E\left[v_{i}\left(\hat{\theta}_{1}\left(\tilde{t}_{1} \mid \delta, w_{n}, n\right), \ldots, \hat{\theta}_{n}\left(\tilde{t}_{n} \mid \delta, w_{n}, n\right) \mid \tilde{t}_{1}, \ldots, \tilde{t}_{n}, \delta, w_{n}, n\right) \mid \tilde{t}_{i}\right]=E \tilde{\theta}_{i}-K$

From (40), in combination with (36) and (37), we further conclude that

$$
\lim _{n \rightarrow \infty} \frac{w_{n}}{2} E\left[\left(\hat{\theta}_{i}\left(\tilde{t}_{i}\right)-\frac{1}{n-2} \sum_{j \neq i, i+1} \tilde{s}_{i}^{j}\right)^{2} \mid \tilde{t}_{i}\right]=0
$$

almost surely, and hence, that

$$
\lim _{n \rightarrow \infty} \frac{w_{n}}{2} E\left[\left(\hat{\theta}_{i}\left(\tilde{t}_{i}\right)-\frac{1}{n-2} \sum_{j \neq i, i+1} \tilde{s}_{i}^{j}\right)^{2}\right]=0 .
$$

Because we may replace $i$ by $i-1$ in (41), we also have

$$
\lim _{n \rightarrow \infty} \frac{w_{n}}{2} E\left[\left(\hat{\theta}_{i-1}\left(\tilde{t}_{i-1}\right)-\frac{1}{n-2} \sum_{j \neq i-1, i} \tilde{s}_{i-1}^{j}\right)^{2}\right]=0 .
$$

Because the term under the expectations operator is everywhere nonnegative, we can also infer that

$$
\lim _{n \rightarrow \infty} \frac{w_{n}}{2} E\left[\left(\hat{\theta}_{i-1}\left(\tilde{t}_{i-1}\right)-\frac{1}{n-2} \sum_{j \neq i-1, i} \tilde{s}_{i-1}^{j}\right)^{2} \mid \tilde{t}_{i}\right]=0,
$$


almost surely. Now (30) follows from (40) and (43).

Theorem 3.3 is the main result of our paper. It shows that, if $n$ is large, the public good is provided with a probability close to one, as required for efficiency. Interim expected payoffs are approximately equal to the expected per capita surplus. Information rents of individuals are approximately equal to zero. Given that expected per capita surplus is strictly positive, interim individual rationality is not an issue.

These findings run counter to the main result in Neeman (2004). The difference is due to the fact that he imposes a uniformity condition on the failure of BDP that is problematic when there are many agents. His uniformity condition leaves no room for the possibility that, in a large economy, with many other agents providing information about agent $i$, agent $i$ may be informationally small.

\section{Discussion: Informational Smallness and BDP}

To conclude the paper, we return to a general discussion of informational smallness and the BDP property. In contrast to McLean and Postlewaite (2002), we have not actually given a formal definition of informational smallness. We have simply worked with a specification in which agents $2, \ldots, n$ have noisy signals $\tilde{s}_{1}^{2}, \ldots, \tilde{s}_{1}^{n}$ about the payoff parameter $\tilde{\theta}_{1}$ of agent 1 , so that, if $n$ is large, the cross-section average $\frac{1}{n-2} \sum_{j=3}^{n} \tilde{s}_{1}^{j}$ provides a fairly precise estimate of $\tilde{\theta}_{1}$, so that a scoring rule can provide agent 1 with an incentive to be close to honest in what he reports about $\tilde{\theta}_{1}$ without his being exposed to too much of a risk from the noise in the other agents' signals.

In contrast to our approach, which focusses on the extent to which the information available to any one agent can be recovered from the information available to the other agents, the definition of informational smallness in McLean and Postlewaite (2002) is concerned with the conditional probability distribution of the overall state of the economy given the information available to the different participants: An agent is informationally small, if, with a probability close to one, the information available to him has only a small effect on the conditional distribution of the overall state of the econ- 
omy. In their model of a pure-exchange economy with common values, the overall state of the economy corresponds to the vector of the common-value parameters for the different goods.

In our analysis of public-good provision under participation constraints, the overall state of the economy would correspond to the vector $\left(\tilde{\theta}_{1}, \ldots, \tilde{\theta}_{n}\right)$ of the different agents' payoff parameters. ${ }^{4}$ Given that agent $i$ knows $\tilde{\theta}_{i}$, the $i$-th element of this vector, and the other agents have only noisy signals about $\tilde{\theta}_{i}$, it is not clear what the precise analogue of the McLean-Postlewaite definition of informational smallness in our setting would be. The issue is compounded by the fact that their definition makes essential use of their assumption that the set of overall states of the economy is finite; in our analysis, the set of possible realizations of the vector $\left(\tilde{\theta}_{1}, \ldots, \tilde{\theta}_{n}\right)$ is only required to be compact. Development of a formal definition of informational smallness that is quite generally applicable in a wide variety of models remains a task of its own. The task is particularly challenging if one wants to go beyond the common-prior abstract type space formulation considered here and to think about the matter in a setting involving a universal type space without a common-prior assumption.

McLean and Postlewaite (2002) actually assume that, within their finitestate model, the BDP property holds, ${ }^{5}$ and they use Crémer-McLean-type bets to ensure incentive compatibility. As they present their results, informational smallness ensures that these bets can be small and, therefore, that they do not cause problems with risk aversion and/or wealth constraints. Given their reliance on the BDP property though, their analysis, like that of Crémer and McLean (1988) is subject to the criticism of Neeman (2004) or Heifetz and Neeman (2006).

By contrast, we do not assume the BDP property. Indeed, we have introduced the signals $\tilde{\sigma}_{j}^{i}$ that agent $i$ receives about the signals $\tilde{s}_{i}^{j}, j \neq i$, for the sole purpose of ensuring that the value of the payoff parameters $\tilde{\theta}_{i}$

\footnotetext{
${ }^{4}$ If we were only concerned with the question of whether the public good is to be provided or not, we might restrict our attention to the cross-section average $\frac{1}{n} \sum \tilde{\theta}_{j}$. However, because of participation constraints, we must consider information about each individual's $\tilde{\theta}_{i}$. Otherwise, it would not be possible to obtain the resources needed for public-good provision.

${ }^{5}$ This is implicit in their treatment of what they call "distributional variability".
} 
cannot be inferred from the agent's beliefs about $\tilde{s}_{i}^{j}, j \neq i$. Our analysis thus shows that informational smallness limits information rents even in the absence of BDP. If the number of agents who have information about each other is large, this effect of informational smallness neutralizes the freerider problem of public-good provision even though the free-rider problem is particularly severe when there are many agents.

There is a sense in which informational smallness implies an approximate BDP property. In our model, the beliefs of agent $i$ about the cross-section mean $\frac{1}{n-1} \sum_{j \neq i} \tilde{s}_{i}^{j}$ are given by a weighted average of his payoff parameter $\tilde{\theta}_{i}$ and the noisy signals $\tilde{\sigma}_{j}^{i}, j \neq i$, that he has received about the signals $\tilde{s}_{i}^{j}, j \neq i$. The weights depend on the noise terms in $\tilde{s}_{i}^{j}$ and $\tilde{\sigma}_{j}^{i}$, but, if $n$ is large, the weight given to $\tilde{\theta}_{i}$ will be close to one, and the sum of the weights that are given to $\tilde{\sigma}_{j}^{i}, j \neq i$, will be close to zero. In other words, we may think of the agent's beliefs about the cross-section mean $\frac{1}{n-1} \sum_{j \neq i} \tilde{s}_{i}^{j}$ as a noisy signal about his payoff parameter $\tilde{\theta}_{i}$, where the noise is small if $n$ is large. In this sense, our model exhibits an approximate BDP property when $n$ is large. ${ }^{6}$

Our implementation result suggests that information rents are small if an approximate BDP property holds. We conjecture that this conclusion holds far beyond the simple example that we have studied, i.e., that, quite generally, the amount of surplus that can be extracted from agents with private information varies continuously with the specification of information even as we move from non-BDP to BDP models. If this conjecture turns out to be correct, it will somewhat defuse the question, treated by Heifetz and Neeman (2006), whether the BDP property and full surplus extraction are "generic" or not. ${ }^{7}$

\footnotetext{
${ }^{6}$ The argument is closely related to the observation of Neeman (2004) that the BDP property encompasses what may be called a collective BDP property, i.e., a situation where the vector of beliefs of all agents "determines" the agent's payoff parameters. This would be the case, e.g., if the information available to agent $i$ is "non-exclusive" in the sense of Postlewaite and Schmeidler (1986), i.e., if this information can be inferred from the information available to all other agents. In such a situation, agent $i$ 's beliefs about the best estimate of $\tilde{\theta}_{i}$ that can be obtained by pooling the other agents' information must be equal to $\tilde{\theta}_{i}$ itself. Thus, BDP must hold.

${ }^{7}$ Heifetz and Neeman (2006) suggest that failures of BDP and therefore also failures of
} 


\section{A Appendix: Proof of Proposition 3.2}

The proof of Proposition 3.2 proceeds in several steps. To simplify the notation, we drop the reference to $\delta, w, n$ as parameters of the payoff functions and strategies whenever this can be done without loss of clarity.

Lemma A.1 A strategy constellation $\left(\hat{t}_{1}(\cdot), \ldots, \hat{t}_{n}(\cdot)\right)$ with $\hat{s}_{j}^{i}\left(t_{i}\right)=s_{j}^{i}$ and $\hat{\sigma}_{j}^{i}\left(t_{i}\right)=\sigma_{j}^{i}$ for all $i$ and all $j \neq i$ is a Bayes-Nash equilibrium if and only if, for all $i$ and all types $t_{i}$ of agent $i$, the report $\hat{\theta}_{i}\left(t_{i}\right)$ satisfies the best-response condition

$$
\begin{aligned}
& E\left[v_{i}\left(\theta_{i}, \hat{\theta}_{1}\left(\tilde{t}_{1}\right), \ldots, \hat{\theta}_{i-1}\left(\tilde{t}_{-1}\right), \hat{\theta}_{i}\left(t_{i}\right), \hat{\theta}_{i+1}\left(\tilde{t}_{i+1}\right), \ldots, \hat{\theta}_{n}\left(\tilde{t}_{n}\right)\right) \mid \tilde{t}_{i}=t_{i}\right] \\
\geq & E\left[v_{i}\left(\theta_{i}, \hat{\theta}_{1}\left(\tilde{t}_{1}\right), \ldots \hat{\theta}_{i-1}\left(\tilde{t}_{i-1}\right), \hat{\theta}_{i}^{\prime}, \hat{\theta}_{i+1}\left(\tilde{t}_{i+1}\right), \ldots, \hat{\theta}_{n}\left(\tilde{t}_{n}\right)\right) \mid \tilde{t}_{i}=t_{i}\right]
\end{aligned}
$$

for all $\hat{\theta}_{i}^{\prime}$, where, as in (35) $v_{i}$ is given by the equation

$$
\begin{aligned}
& v_{i}\left(\hat{\theta}_{1}, \ldots, \hat{\theta}_{n} \mid t_{1}, \ldots, t_{n}\right) \\
= & g\left(\frac{1}{\delta}\left(\frac{1}{n} \sum_{j} \hat{\theta}_{j}-K\right)\right) \times\left(\theta_{i}-\frac{n-1}{n} \hat{\theta}_{i}+\frac{1}{n} \sum_{j \neq i} \hat{\theta}_{j}-K\right) \\
& -\frac{w}{2}\left(\hat{\theta}_{i}-\frac{1}{n-2} \sum_{j \neq i, i+1} s_{i}^{j}\right)^{2} .
\end{aligned}
$$

Proof. The lemma follows directly from the fact that $u_{i}\left(\hat{t}_{1}, \ldots, \hat{t}_{n}\right)$ is independent of $\hat{s}_{j}^{i}$ and $\hat{\sigma}_{j}^{i}, j \neq i$, and that the term $\frac{w}{2}\left(\hat{\theta}_{i-1}-\frac{1}{n-2} \sum_{j \neq i-1, i} s_{i-1}^{j}\right)^{2}$ in $u_{i}\left(\hat{t}_{1}, \ldots, \hat{t}_{n}\right)$ is also independent of $\hat{\theta}_{i}$.

full surplus extraction are generic, where genericity is interpreted in a measure-theoretic sense. Gizatulina and Hellwig (2009) argue that their analysis neglects the fact that, if beliefs are interpreted as conditional expectations, given the available information, an agent's payoff parameter, as one piece of information that is available to him, should be one of the conditioning variables. Relying on a topological concept of genericity, Gizatulina and Hellwig (2009) show that the BDP property is generic if the set of variables about which the agent forms expectations is sufficiently rich. 
Lemma A.2 For any $i$, any type $t_{i}$ of agent $i$, and any reporting strategies $\hat{\theta}_{j}(\cdot)$ of agents $j \neq i$, the best-response condition (44) has a solution $\hat{\theta}_{i}\left(t_{t}\right)$. Moreover, this solution satisfies.

$$
\hat{\theta}_{i}\left(t_{i}\right) \in\left[y\left(t_{i}\right), z\left(t_{i}\right)\right]
$$

where

$$
\begin{gathered}
y\left(t_{i}\right):=\min \left(\theta_{i}, \frac{1}{n-2} E\left[\sum_{j \neq i, i+1} s_{i}^{j} \mid \tilde{t}_{i}=t_{i}\right]-\frac{G^{1}}{w}\right) \\
\text { and } \\
z\left(t_{i}\right):=\max \left(\theta_{i}, \frac{1}{n-2} E\left[\sum_{j \neq i, i+1} s_{i}^{j} \mid \tilde{t}_{i}=t_{i}\right]+\frac{G^{1}}{w}\right) .
\end{gathered}
$$

Proof. To prove this lemma, we compute the slope of the function

$$
\hat{\theta}_{i} \rightarrow E\left[v_{i}\left(\hat{\theta}_{1}\left(\tilde{t}_{1}\right), \ldots, \hat{\theta}_{i-1}\left(\tilde{t}_{i-1}\right), \hat{\theta}_{i}, \hat{\theta}_{i+1}\left(\tilde{t}_{i+1}\right), \ldots, \hat{\theta}_{n}\left(\tilde{t}_{n}\right) \mid \tilde{t}_{1}, \ldots, \tilde{t}_{n}\right) \mid \tilde{t}_{i}=t_{i}\right] .
$$

From (45), we obtain

$$
\begin{aligned}
& \frac{\partial E\left[v_{i}\left(\hat{\theta}_{1}\left(\tilde{t}_{1}\right), \ldots, \hat{\theta}_{n}\left(\tilde{t}_{n}\right) \mid \tilde{t}_{1}, \ldots, \tilde{t}_{n}\right) \mid \tilde{t}_{i}=t_{i}\right]}{\partial \hat{\theta}_{i}} \\
= & E\left[\frac{g^{\prime}}{\delta n} \times\left(\theta_{i}-\frac{n-1}{n} \hat{\theta}_{i}+\frac{1}{n} \sum_{j \neq i} \hat{\theta}_{j}\left(\tilde{t}_{j}\right)-K\right) \mid \tilde{t}_{i}=t_{i}\right] \\
& -\frac{n-1}{n} E\left[g \mid \tilde{t}_{i}=t_{i}\right]-w\left(\hat{\theta}_{i}-\frac{1}{n-2} E\left[\sum_{j \neq i, i+1} s_{i}^{j} \mid \tilde{t}_{i}=t_{i}\right]\right)
\end{aligned}
$$

where both $g^{\prime}$ and $g$ are evaluated at $\frac{1}{\delta}\left(\frac{1}{n}\left(\hat{\theta}_{i}\left(t_{i}\right)+\sum_{k \neq i} \hat{\theta}_{k}\left(\tilde{t}_{k}\right)\right)-K\right)$. 
$\operatorname{By}(11), g^{\prime}\left(\frac{1}{\delta}\left(\frac{1}{n}\left(\hat{\theta}_{i}\left(t_{i}\right)+\sum_{k \neq i} \hat{\theta}_{k}\left(\tilde{t}_{k}\right)\right)-K\right)=0\right.$ if $\frac{1}{n}\left(\hat{\theta}_{i}\left(t_{i}\right)+\sum_{k \neq i} \hat{\theta}_{k}\left(\tilde{t}_{k}\right)\right)-$ $K>\delta$. From (49) and (22), one therefore obtains

$$
\begin{aligned}
& \frac{\partial E\left[v_{i}\left(\hat{\theta}_{1}\left(\tilde{t}_{1}\right), \ldots, \hat{\theta}_{n}\left(\tilde{t}_{n}\right) \mid \tilde{t}_{1}, \ldots, \tilde{t}_{n}\right) \mid \tilde{t}_{i}=t_{i}\right]}{\partial \hat{\theta}_{i}}\left(\hat{\theta}_{i}\right) \\
\leq & E \frac{g^{\prime}}{\delta n} \times\left(\theta_{i}-\hat{\theta}_{i}\right)+\frac{G^{1}}{n} \\
& -w\left(\hat{\theta}_{i}-\frac{1}{n-2} E\left[\sum_{j \neq i, i+1} s_{i}^{j} \mid \tilde{t}_{i}=t_{i}\right]\right) .
\end{aligned}
$$

Thus,

$$
\frac{\partial E\left[v_{i}\left(\hat{\theta}_{1}\left(\tilde{t}_{1}\right), \ldots, \hat{\theta}_{n}\left(\tilde{t}_{n}\right) \mid \tilde{t}_{1}, \ldots, \tilde{t}_{n}\right) \mid \tilde{t}_{i}=t_{i}\right]}{\partial \hat{\theta}_{i}}\left(\hat{\theta}_{i}\right)<0
$$

if

$$
\hat{\theta}_{i}>\theta_{i} \text { and } \hat{\theta}_{i}>\frac{1}{n-2} E\left[\sum_{j \neq i, i+1} s_{i}^{j} \mid \tilde{t}_{i}=t_{i}\right]+\frac{G^{1}}{w n} .
$$

In particular, $E\left[v_{i}\left(\hat{\theta}_{1}\left(\tilde{t}_{1}\right), \ldots, \hat{\theta}_{n}\left(\tilde{t}_{n}\right) \mid \tilde{t}_{1}, \ldots, \tilde{t}_{n}\right) \mid \tilde{t}_{i}=t_{i}\right]$ is decreasing in $\hat{\theta}_{i}$ if $\hat{\theta}_{i}>z\left(t_{i}\right)$ where $z\left(t_{i}\right)$ is given by $(47)$.

By $(11)$, one also has $g^{\prime}\left(\frac{1}{\delta}\left(\frac{1}{n}\left(\hat{\theta}_{i}\left(t_{i}\right)+\sum_{k \neq i} \hat{\theta}_{k}\left(\tilde{t}_{k}\right)\right)-K\right)=0\right.$ if $\frac{1}{n}\left(\hat{\theta}_{i}\left(t_{i}\right)+\right.$ $\left.\sum_{k \neq i} \hat{\theta}_{k}\left(\tilde{t}_{k}\right)\right)-K<0$. From (49) and (22) and the fact that $g$ takes values between zero and one, one therefore obtains

$$
\begin{aligned}
& \frac{\partial E\left[v_{i}\left(\hat{\theta}_{1}\left(\tilde{t}_{1}\right), \ldots, \hat{\theta}_{n}\left(\tilde{t}_{n}\right) \mid \tilde{t}_{1}, \ldots, \tilde{t}_{n}\right) \mid \tilde{t}_{i}=t_{i}\right]}{\partial \hat{\theta}_{i}}\left(\hat{\theta}_{i}\right) \\
\geq & E \frac{g^{\prime}}{\delta n} \times\left(\theta_{i}-\hat{\theta}_{i}\right)-1 \\
& -w\left(\hat{\theta}_{i}-\frac{1}{n-1} E\left[\sum_{j \neq i} s_{i}^{j} \mid \tilde{t}_{i}=t_{i}\right]\right) .
\end{aligned}
$$

It follows that

$$
\frac{\partial E\left[v_{i}\left(\hat{\theta}_{1}\left(\tilde{t}_{1}\right), \ldots, \hat{\theta}_{n}\left(\tilde{t}_{n}\right) \mid \tilde{t}_{1}, \ldots, \tilde{t}_{n}\right) \mid \tilde{t}_{i}=t_{i}\right]}{\partial \hat{\theta}_{i}}\left(\hat{\theta}_{i}\right)>0
$$


if

$$
\hat{\theta}_{i}<\theta_{i} \text { and } \hat{\theta}_{i}<\frac{1}{n-2} E\left[\sum_{j \neq i, i+1} s_{i}^{j} \mid \tilde{t}_{i}=t_{i}\right]-\frac{1}{w} .
$$

Since $G^{1}>1$, by $(23)$, it follows, in particular, that $E\left[v_{i}\left(\hat{\theta}_{1}\left(\tilde{t}_{1}\right), \ldots, \hat{\theta}_{n}\left(\tilde{t}_{n}\right) \mid \tilde{t}_{1}, \ldots, \tilde{t}_{n}\right) \mid \tilde{t}_{i}=t_{i}\right]$ is increasing in $\hat{\theta}_{i}$ if $\hat{\theta}_{i}<y\left(t_{i}\right)$, where $y\left(t_{i}\right)$ is given by (46).

Because the function

$$
\hat{\theta}_{i} \rightarrow E\left[v_{i}\left(\hat{\theta}_{1}\left(\tilde{t}_{1}\right), \ldots, \hat{\theta}_{i-1}\left(\tilde{t}_{-1}\right), \hat{\theta}_{i}, \hat{\theta}_{i+1}\left(\tilde{t}_{i+1}\right), \ldots, \hat{\theta}_{n}\left(\tilde{t}_{n}\right) \mid \tilde{t}_{1}, \ldots, \tilde{t}_{n}\right) \mid \tilde{t}_{i}=t_{i}\right]
$$

is continuous, it has a maximum on the compact interval $\left[y\left(t_{i}\right), z\left(t_{i}\right)\right]$. Because this function is increasing below $y\left(t_{i}\right)$ and decreasing above $z\left(t_{i}\right)$, this maximum is actually a global maximum of the function (48). The proof of Lemma A.2 is thereby complete.

REMARK A.3 For $\hat{\theta}_{i}$ belonging to the interval $\left[y\left(t_{i}\right), z\left(t_{i}\right)\right]$ given by (46), (47),

$$
\left|\theta_{i}-\hat{\theta}_{i}\right| \leq \bar{\theta}+\bar{s}+\frac{G^{1}}{w}
$$

where, again, $\bar{\theta}$ and $\bar{s}$ are such that $|\theta| \leq \bar{\theta}$ for all $\theta \in \Theta$ and $|s| \leq \bar{s}$ for all $s \in S$.

Proof. If $\theta_{i}>\hat{\theta}_{i}$ and $\hat{\theta}_{i}$ belongs to $\left[y\left(t_{i}\right), z\left(t_{i}\right)\right]$, one has $\theta_{i}>\hat{\theta}_{i}>$ $\frac{1}{n-2} E\left[\sum_{j \neq i, i+1} s_{i}^{j} \mid \tilde{t}_{i}=t_{i}\right]-\frac{G^{1}}{w} \geq-\bar{s}-\frac{G^{1}}{w}$, hence $\left|\theta_{i}-\hat{\theta}_{i}\right| \leq \bar{\theta}+\bar{s}+\frac{G^{1}}{w}$. If $\theta_{i}<\hat{\theta}_{i}$ and $\hat{\theta}_{i}$ belongs to (50), a similar computation yields $\left|\theta_{i}-\hat{\theta}_{i}\right| \leq \bar{\theta}+\bar{s}+$ $\frac{G^{1}}{w}$.

Lemma A.4 If condition (24) holds, then, for any $i$, any type $t_{i}$ of agent $i$, and any reporting strategies $\hat{\theta}_{j}(\cdot)$ of agents $j \neq i$, the best response $\hat{\theta}_{i}\left(t_{t}\right)$ of agent $i$ to the strategies $\hat{\theta}_{j}(\cdot), j \neq i$, is unique. 
Proof. To prove this assertion, we compute the second derivative

$$
\begin{aligned}
& \frac{\partial^{2} E\left[v_{i}\left(\hat{\theta}_{1}\left(\tilde{t}_{1}\right), \ldots, \hat{\theta}_{n}\left(\tilde{t}_{n}\right) \mid \tilde{t}_{1}, \ldots, \tilde{t}_{n}\right) \mid \tilde{t}_{i}=t_{i}\right]}{\partial \hat{\theta}_{i}^{2}} \\
= & E\left[\frac{g^{\prime \prime}}{\delta^{2} n^{2}} \times\left(\theta_{i}-\frac{n-1}{n} \hat{\theta}_{i}+\frac{1}{n} \sum_{j \neq i} \hat{\theta}_{j}\left(\tilde{t}_{j}\right)-K\right) \mid \tilde{t}_{i}=t_{i}\right] \\
& -2 \frac{n-1}{\delta n^{2}} E\left[g^{\prime} \mid \tilde{t}_{i}=t_{i}\right]-w,
\end{aligned}
$$

where $g^{\prime \prime}$ and $g^{\prime}$ are evaluated at $\frac{1}{\delta}\left(\frac{1}{n}\left(\hat{\theta}_{i}\left(t_{i}\right)+\sum_{k \neq i} \hat{\theta}_{k}\left(\tilde{t}_{k}\right)\right)-K\right)$. By (11), $g^{\prime \prime}\left(\frac{1}{\delta}\left(\frac{1}{n}\left(\hat{\theta}_{i}\left(t_{i}\right)+\sum_{k \neq i} \hat{\theta}_{k}\left(\tilde{t}_{k}\right)\right)-K\right)=0\right.$ if $\frac{1}{n}\left(\hat{\theta}_{i}\left(t_{i}\right)+\sum_{k \neq i} \hat{\theta}_{k}\left(\tilde{t}_{k}\right)\right)-K>\delta$. From (51) and (22), one therefore obtains

$$
\frac{\partial^{2} E\left[v_{i}\left(\hat{\theta}_{1}\left(\tilde{t}_{1}\right), \ldots, \hat{\theta}_{n}\left(\tilde{t}_{n}\right) \mid \tilde{t}_{1}, \ldots, \tilde{t}_{n}\right) \mid \tilde{t}_{i}=t_{i}\right]}{\partial \hat{\theta}_{i}^{2}} \leq \frac{G^{2}}{\delta^{2} n^{2}}\left(\left|\theta_{i}-\hat{\theta}_{i}\right|+\delta\right)-w .
$$

By Remark A.3, it follows that

$$
\frac{\partial^{2} E\left[v_{i}\left(\hat{\theta}_{1}\left(\tilde{t}_{1}\right), \ldots, \hat{\theta}_{n}\left(\tilde{t}_{n}\right) \mid \tilde{t}_{1}, \ldots, \tilde{t}_{n}\right) \mid \tilde{t}_{i}=t_{i}\right]}{\partial \hat{\theta}_{i}^{2}} \leq \frac{G^{2}}{\delta^{2}}\left(\bar{\theta}+\bar{s}+\frac{G^{1}}{w}+\delta\right)-w .
$$

If condition (24) holds, the right-hand side of (53) is negative. In this case, the function $E\left[v_{i}\left(\hat{\theta}_{1}\left(\tilde{t}_{1}\right), \ldots, . \hat{\theta}_{i-1}\left(\tilde{t}_{-1}\right), \cdot, \hat{\theta}_{i+1}\left(\tilde{t}_{i+1}\right), \ldots, \hat{\theta}_{n}\left(\tilde{t}_{n}\right) \mid \tilde{t}_{1}, \ldots, \tilde{t}_{n}\right) \mid \tilde{t}_{i}=t_{i}\right]$ is strictly concave and has a unique maximum on the interval (50). By the argument given in the proof of Lemma A.2 this is also the unique global maximum.

Lemma A.5 If condition (24) holds, then, for any $i$, any type $t_{i}$ of agent $i$, and any reporting strategies $\hat{\theta}_{j}(\cdot)$ of agents $j \neq i, \hat{\theta}_{i}\left(t_{i}\right)$ is a best response of agent $i$ to the strategies $\hat{\theta}_{j}(\cdot), j \neq i$, if and only if condition (25) is satisfied.

Proof. By (49), (25) is just the first-order condition for $\hat{\theta}_{i}\left(t_{i}\right)$. Lemma A.4 implies that, if condition (24) holds, the first-order condition is sufficient as well as necessary for $\hat{\theta}_{i}\left(t_{i}\right)$ to be a best response of agent $i$ to the strategies $\hat{\theta}_{j}(\cdot), j \neq i$. 
Lemma A.6 If condition (24) holds, there exists a unique vector of functions $\hat{\theta}_{k}(\cdot), k=1, \ldots, n$ such that condition (25) holds for all $i$ and all types $t_{i}$ of agent $i$.

Proof. To prove this assertion, we use a contraction mapping argument. Rewrite (25) in the equivalent form

$$
\begin{aligned}
\hat{\theta}_{i}\left(t_{i}\right)= & \frac{1}{n-2} \sum_{j \neq i, i+1} E\left[s_{i}^{j} \mid \tilde{t}_{i}=t_{i}\right] \\
& +\frac{1}{w} E\left[\frac{g^{\prime}}{n \delta} \times\left(\theta_{i}-\frac{n-1}{n} \hat{\theta}_{i}\left(\tilde{t}_{i}\right)+\frac{1}{n} \sum_{k \neq i} \hat{\theta}_{k}\left(\tilde{t}_{k}\right)-K\right) \mid \tilde{t}_{i}=t_{i}\right] \\
& -\frac{1}{w} \frac{n-1}{n} E\left[g \mid \tilde{t}_{i}=t_{i}\right] .
\end{aligned}
$$

Equations (54) for $i=1, \ldots, n$ can be treated as a system of functional equations for $\hat{\theta}_{i}(\cdot), i=1, \ldots, n$. A solution $\hat{\theta}_{i}(\cdot), i=1, \ldots, n$ to this system of functional equations must be a fixed point of the mapping $T$ that maps any vector $\hat{\boldsymbol{\theta}}=\left(\hat{\theta}_{1}(\cdot), \ldots, \hat{\theta}_{n}(\cdot)\right)$ of real-valued measurable functions on $\Theta \times S^{n-1} \times \mathbb{R}^{n-1}$ into a vector $T \hat{\boldsymbol{\theta}}=\left(T_{1} \hat{\boldsymbol{\theta}}, \ldots, T_{n} \hat{\boldsymbol{\theta}}\right)$ of real-valued measurable functions on $\Theta \times S^{n-1} \times \mathbb{R}^{n-1}$ such that, for any $i$ and any $t_{i} \in \Theta \times S^{n-1} \times \mathbb{R}^{n-1}$,

$$
\begin{aligned}
\left(T_{i} \hat{\boldsymbol{\theta}}\right)\left(t_{i}\right)= & \frac{1}{n-2} \sum_{j \neq i, i+1} E\left[s_{i}^{j} \mid \tilde{t}_{i}=t_{i}\right] \\
& +\frac{1}{w} E\left[\frac{g^{\prime}}{n \delta} \times\left(\theta_{i}-\hat{\theta}_{i}\left(\tilde{t}_{i}\right)+\frac{1}{n} \sum_{k} \hat{\theta}_{k}\left(\tilde{t}_{k}\right)-K\right) \mid \tilde{t}_{i}=t_{i}\right] \\
& -\frac{1}{w} \frac{n-1}{n} E\left[g \mid \tilde{t}_{i}=t_{i}\right],
\end{aligned}
$$

where, as before, $g^{\prime}$ and $g$ are evaluated at $\frac{1}{\delta}\left(\frac{1}{n}\left(\hat{\theta}_{i}\left(t_{i}\right)+\sum_{k \neq i} \hat{\theta}_{k}\left(\tilde{t}_{k}\right)\right)-K\right)$.

By Lemma A.2, for any vector $\hat{\boldsymbol{\theta}}=\left(\hat{\theta}_{1}(\cdot), \ldots, \hat{\theta}_{n}(\cdot)\right)$ of real-valued measurable functions on $\Theta \times S^{n-1} \times \mathbb{R}^{n-1}$ the functions $\left(T_{i} \hat{\boldsymbol{\theta}}\right)(\cdot)$ take values in the interval (50). We can therefore think of $T$ as mapping vectors of bounded real-valued measurable functions on $\Theta \times S^{n-1} \times \mathbb{R}^{n-1}$ into vectors of bounded real-valued measurable functions on $\Theta \times S^{n-1} \times \mathbb{R}^{n-1}$. When endowed with the metric

$$
\rho(\hat{\boldsymbol{\theta}}, \overline{\boldsymbol{\theta}})=\max _{i} \sup _{t_{i} \in \Theta \times S^{n-1} \times \mathbb{R}^{n-1}}\left|\hat{\theta}_{i}\left(t_{i}\right)-\bar{\theta}_{i}\left(t_{i}\right)\right|,
$$


the space of bounded real-valued measurable functions on $\Theta \times S^{n-1} \times \mathbb{R}^{n-1}$ is a complete metric space (Dunford and Schwartz (1988), p.258).

We want to show that, for some $\alpha<1$,

$$
\rho(T \hat{\boldsymbol{\theta}}, T \overline{\boldsymbol{\theta}}) \leq \alpha \rho(\hat{\boldsymbol{\theta}}, \overline{\boldsymbol{\theta}})
$$

if $w$ satisfies condition (24). For this purpose, we use (55) to compute

$$
\begin{aligned}
\left(T_{i} \hat{\boldsymbol{\theta}}\right)\left(t_{i}\right)-\left(T_{i} \overline{\boldsymbol{\theta}}\right)\left(t_{i}\right)= & \frac{1}{w} E\left[\frac{g^{\prime}}{n \delta} \times\left(\theta_{i}-\hat{\theta}_{i}\left(\tilde{t}_{i}\right)+\frac{1}{n} \sum_{k} \hat{\theta}_{k}\left(\tilde{t}_{k}\right)-K\right) \mid \tilde{t}_{i}=t_{i}\right] \\
& -\frac{1}{w} E\left[\frac{\bar{g}^{\prime}}{n \delta} \times\left(\theta_{i}-\bar{\theta}_{i}\left(\tilde{t}_{i}\right)+\frac{1}{n} \sum_{k} \bar{\theta}_{k}\left(\tilde{t}_{k}\right)-K\right) \mid \tilde{t}_{i}=t_{i}\right] \\
& +\frac{1}{w} \frac{n-1}{n}\left(E\left[g \mid \tilde{t}_{i}=t_{i}\right]-E\left[\bar{g} \mid \tilde{t}_{i}=t_{i}\right]\right)
\end{aligned}
$$

where, in the first and third terms, $g^{\prime}$ and $g$ are evaluated at $\frac{1}{\delta}\left(\frac{1}{n}\left(\sum_{k} \hat{\theta}_{k}\left(\tilde{t}_{k}\right)\right)-K\right)$, as before, and the symbols $\bar{g}^{\prime}$ and $\bar{g}$ in the second and third terms refer to $g^{\prime}$ and $g$ evaluated at $\frac{1}{\delta}\left(\frac{1}{n}\left(\sum_{k} \bar{\theta}_{k}\left(\tilde{t}_{k}\right)\right)-K\right)$. For the third term in $(57)$, the mean-value theorem yields

$$
\begin{aligned}
& \left|\frac{1}{w} \frac{n-1}{n}\left(E\left[g \mid \tilde{t}_{i}=t_{i}\right]-E\left[\bar{g} \mid \tilde{t}_{i}=t_{i}\right]\right)\right| \\
= & \frac{1}{w} \frac{n-1}{n} E\left[g^{\prime \prime} \frac{1}{\delta n}\left[\sum_{k} \hat{\theta}_{k}\left(\tilde{t}_{k}\right)-\sum_{k} \bar{\theta}_{k}\left(\tilde{t}_{k}\right)\right] \mid \tilde{t}_{i}=t_{i}\right],
\end{aligned}
$$

where $g^{\prime \prime}$ is evaluated at some point between $\frac{1}{\delta}\left(\frac{1}{n}\left(\sum_{k} \hat{\theta}_{k}\left(\tilde{t}_{k}\right)\right)-K\right)$ and $\frac{1}{\delta}\left(\frac{1}{n}\left(\sum_{k} \bar{\theta}_{k}\left(\tilde{t}_{k}\right)\right)-K\right)$. Therefore,

$$
\begin{aligned}
\left|\frac{1}{w} \frac{n-1}{n}\left(E\left[g \mid \tilde{t}_{i}=t_{i}\right]-E\left[\bar{g} \mid \tilde{t}_{i}=t_{i}\right]\right)\right| & \leq \frac{1}{w} \frac{n-1}{n} \frac{G^{2}}{\delta n}\left[\sum_{k}\left|\hat{\theta}_{k}\left(\tilde{t}_{k}\right)-\bar{\theta}_{k}\left(\tilde{t}_{k}\right)\right|\right] \\
& \leq \frac{1}{w} \frac{G^{2}}{\delta} \rho(\hat{\boldsymbol{\theta}}, \overline{\boldsymbol{\theta}}) .
\end{aligned}
$$

As for the first and second terms in (57), it is convenient to write them as

$$
\begin{gathered}
\frac{1}{w} E\left[\frac{g^{\prime}}{n \delta} \times\left(\theta_{i}-\hat{\theta}_{i}\left(\tilde{t}_{i}\right)\right) \mid \tilde{t}_{i}=t_{i}\right]-\frac{1}{w} E\left[\frac{\bar{g}^{\prime}}{n \delta} \times\left(\theta_{i}-\bar{\theta}_{i}\left(\tilde{t}_{i}\right)\right) \mid \tilde{t}_{i}=t_{i}\right] \\
+\frac{1}{w} E\left[\frac{g^{\prime}}{n \delta} \times\left(\frac{1}{n} \sum_{k} \hat{\theta}_{k}\left(\tilde{t}_{k}\right)-K\right) \mid \tilde{t}_{i}=t_{i}\right]-\frac{1}{w} E\left[\frac{\bar{g}^{\prime}}{n \delta} \times\left(\frac{1}{n} \sum_{k} \bar{\theta}_{k}\left(\tilde{t}_{k}\right)-K\right) \mid \tilde{t}_{i}=t_{i}\right] .
\end{gathered}
$$


For the first of these differences, we compute

$$
\begin{aligned}
& \left|\frac{1}{w} E\left[\frac{g^{\prime}}{n \delta} \times\left(\theta_{i}-\hat{\theta}_{i}\left(\tilde{t}_{i}\right)\right) \mid \tilde{t}_{i}=t_{i}\right]-\frac{1}{w} E\left[\frac{\bar{g}^{\prime}}{n \delta} \times\left(\theta_{i}-\bar{\theta}_{i}\left(\tilde{t}_{i}\right)\right) \mid \tilde{t}_{i}=t_{i}\right]\right| \\
\leq & \frac{1}{w} E\left[\frac{g^{\prime}-\bar{g}^{\prime}}{n \delta} \times\left(\theta_{i}-\hat{\theta}_{i}\left(\tilde{t}_{i}\right)\right) \mid \tilde{t}_{i}=t_{i}\right]+\frac{1}{w} \frac{G^{1}}{n \delta} \times\left|\bar{\theta}_{i}\left(\tilde{t}_{i}\right)-\hat{\theta}_{i}\left(\tilde{t}_{i}\right)\right| .
\end{aligned}
$$

By Lemma A.2 and Remark A.3, we may suppose that $\left|\theta_{i}-\hat{\theta}_{i}\left(t_{i}\right)\right| \leq \bar{\theta}+\bar{s}+$ $\frac{G^{1}}{w}$. By the mean-value theorem and (22), we also have $g^{\prime}-\bar{g}^{\prime} \leq \frac{G^{2}}{n \delta} n \rho(\hat{\boldsymbol{\theta}}, \overline{\boldsymbol{\theta}})$. Therefore we can write

$$
\begin{gathered}
\left|\frac{1}{w} E\left[\frac{g^{\prime}}{n \delta} \times\left(\theta_{i}-\hat{\theta}_{i}\left(\tilde{t}_{i}\right)\right) \mid \tilde{t}_{i}=t_{i}\right]-\frac{1}{w} E\left[\frac{\bar{g}^{\prime}}{n \delta} \times\left(\theta_{i}-\bar{\theta}_{i}\left(\tilde{t}_{i}\right)\right) \mid \tilde{t}_{i}=t_{i}\right]\right| \\
\leq \frac{1}{w}\left[\left(\bar{\theta}+\bar{s}+\frac{G^{1}}{w}\right) \frac{G^{2}}{n \delta^{2}}+\frac{G^{1}}{n \delta}\right] \rho(\hat{\boldsymbol{\theta}}, \overline{\boldsymbol{\theta}}) .
\end{gathered}
$$

Turning to the second of the differences in (59), we note that, if neither $\frac{1}{n} \sum_{k} \hat{\theta}_{k}\left(\tilde{t}_{k}\right)-K$ nor $\frac{1}{n} \sum_{k} \bar{\theta}_{k}\left(\tilde{t}_{k}\right)-K$ lies in the interval $(0, \delta)$, then, by (11), this difference is just zero. Suppose, therefore, that $\frac{1}{n} \sum_{k} \hat{\theta}_{k}\left(\tilde{t}_{k}\right)-K \in(0, \delta)$ and write

$$
\begin{aligned}
& \frac{1}{w} E\left[\frac{g^{\prime}}{n \delta} \times\left(\frac{1}{n} \sum_{k} \hat{\theta}_{k}\left(\tilde{t}_{k}\right)-K\right) \mid \tilde{t}_{i}=t_{i}\right]-\frac{1}{w} E\left[\frac{\bar{g}^{\prime}}{n \delta} \times\left(\frac{1}{n} \sum_{k} \bar{\theta}_{k}\left(\tilde{t}_{k}\right)-K\right) \mid \tilde{t}_{i}=t_{i}\right] \\
\leq & \frac{1}{w} E\left[\frac{\left|g^{\prime}-\bar{g}^{\prime}\right|}{n \delta} \times\left(\frac{1}{n} \sum_{k} \hat{\theta}_{k}\left(\tilde{t}_{k}\right)-K\right) \mid \tilde{t}_{i}=t_{i}\right] \\
& +\frac{1}{w} \frac{1}{n} E\left[\frac{\bar{g}^{\prime}}{n \delta} \times\left|\sum_{k} \hat{\theta}_{k}\left(\tilde{t}_{k}\right)-\bar{\theta}_{k}\left(\tilde{t}_{k}\right)\right| \mid \tilde{t}_{i}=t_{i}\right] \\
\leq & \frac{1}{w} \delta \frac{G^{2}}{n^{2} \delta^{2}} n \rho(\hat{\boldsymbol{\theta}}, \overline{\boldsymbol{\theta}})+\frac{1}{w} \frac{1}{n} \frac{G^{1}}{n \delta} n \rho(\hat{\boldsymbol{\theta}}, \overline{\boldsymbol{\theta}})=\frac{1}{w}\left[\frac{G^{2}}{n \delta}+\frac{G^{1}}{n \delta}\right] \rho(\hat{\boldsymbol{\theta}}, \overline{\boldsymbol{\theta}})
\end{aligned}
$$

From (57) - (62), we now find that, for any $\hat{\boldsymbol{\theta}}$ and $\overline{\boldsymbol{\theta}}$ and any $i$ and $t_{i}$,

$$
\begin{aligned}
\left|\left(T_{i} \hat{\boldsymbol{\theta}}\right)\left(t_{i}\right)-\left(T_{i} \overline{\boldsymbol{\theta}}\right)\left(t_{i}\right)\right| & \leq \frac{1}{w}\left[\left(\bar{\theta}+\bar{s}+\frac{G^{1}}{w}\right) \frac{G^{2}}{n \delta^{2}}+\frac{G^{1}}{n \delta}+\frac{G^{2}}{n \delta}+\frac{G^{1}}{n \delta}+\frac{G^{2}}{\delta}\right] \rho(\hat{\boldsymbol{\theta}}, \overline{\boldsymbol{\theta}}) \\
& \leq \frac{1}{w}\left[\left(\bar{\theta}+\bar{s}+\frac{G^{1}}{w}+2 \delta\right) \frac{G^{2}}{\delta^{2}}+2 \frac{G^{1}}{\delta}\right] \rho(\hat{\boldsymbol{\theta}}, \overline{\boldsymbol{\theta}}),
\end{aligned}
$$

hence

$$
\rho(T \hat{\boldsymbol{\theta}}, T \overline{\boldsymbol{\theta}}) \leq \frac{1}{w}\left[\left(\bar{\theta}+\bar{s}+\frac{G^{1}}{w}+2 \delta\right) \frac{G^{2}}{\delta^{2}}+2 \frac{G^{1}}{\delta}\right] \rho(\hat{\boldsymbol{\theta}}, \overline{\boldsymbol{\theta}})
$$


If (24) holds, the factor $\frac{1}{w}\left[\left(\bar{\theta}+\bar{s}+\frac{G^{1}}{w}+2 \delta\right) \frac{G^{2}}{\delta^{2}}+2 \frac{G^{1}}{\delta}\right]$ in (63) is strictly less than one, which proves that, in this case, $T$ is indeed a contraction mapping.

By Banach's fixed-point theorem (see, e.g., Kolmogorov and Fomin (1970), p.67), it follows that $T$ has a unique fixed point $\hat{\boldsymbol{\theta}}=\left(\hat{\theta}_{1}(\cdot), \ldots, \hat{\theta}_{n}(\cdot)\right)$. This fixed point provides the unique solution to the system of functional equations (54) or, equivalently, (25). By Lemma A.5, for each $i$, the function $\hat{\theta}_{i}(\cdot)$ gives the best-response strategy of agent $i$ to the strategies $\hat{\theta}_{j}(\cdot), j \neq i$, of agents other than $i$. This completes the proof of the proposition.

\section{References}

Crémer, J., and R. McLean (1988): "Full extraction of the surplus in Bayesian and dominant strategy auctions," Econometrica, 56, 1247-1257.

Doob, J. (1953): Stochastic Processes. Wiley Interscience, New York.

Dunford, N., and J. Schwartz (1988): Linear Operators, General Theory. Wiley Interscience.

Gizatulina, A., and M. Hellwig (2009): "Payoffs could be inferred from beliefs, generically, when beliefs are conditioned on information," Working paper, Max-Planck Institute for Research on Collective Goods.

Gul, F., And A. Postlewaite (1992): "Asymptotic Efficiency in Large Exchange Economies with Asymmetric Information," Econometrica, 60, 1273-1292.

Heifetz, A., and Z. Neeman (2006): "On the generic (im)possibility of full surplus extraction in mechanism design," Econometrica, 117, 213-233.

Kolmogorov, A., And S. Fomin (1970): Introductory Real Analysis. Prentice-Hall, Englewood Cliff.

Mailath, G., And A. Postlewaite (1990): "Asymmetric information bargaining problems with many agents," Review of Economic Studies, 57, $351-367$. 
McAfee, P., and P. Reny (1992): "Correlated information and mechnanism design," Econometrica, 60, 395-421.

Mclean, R., and A. Postlewaite (2002): "Informational size and incentive compatibility," Econometrica, 70, 2421-2453.

Miller, N., J. Pratt, R. Zeckhauser, and S. Johnson (2007): "Mechanism design with multidimensional, continuous types and interdependent valuations," Journal of Economic Theory, 136, 476-496.

NeEman, Z. (2004): "The relevance of private information in mechanism design," Journal of Economic Theory, 117, 55-77.

Palfrey, T., and S. Srivastava (1986): "Private Information in Large Economies," Journal of Economic Theory, 39, 34 - 58.

Postlewaite, A., And D. Schmeidler (1986): "Implementation in Differential Information Economies," Journal of Economic Theory, 39, 14 33. 


\section{Preprints 2009}

2009/27: Hahmeier M., Prices versus Quantities in Electricity Generation

2009/26: Burhop C., The Transfer of Patents in Imperial Germany

2009/25: Burhop C., Lübbers T., The Historical Market for Technology Licenses: Chemicals, Pharmaceuticals, and Electrical Engineering in Imperial Germany

2009/24: Engel C., Competition as a Socially Desirable Dilemma Theory vs. Experimental Evidence

2009/23: Morell A., Glöckner A., Towfigh E., Sticky Rebates: Rollback Rebates Induce Non-Rational Loyalty in Consumers Experimental Evidence

2009/22: Traxler C., Majority Voting and the Welfare Implications of Tax Avoidance

2009/21: Beckenkamp M., Engel C., Glöckner A., Irlenbusch B., Hennig-Schmidt H., Kube S., Kurschilgen M., Morell A., Nicklisch A., Normann H., Towfigh E., Beware of Broken Windows! First Impressions in Public-good Experiments

2009/20: Nikiforakis N., Normann H., Wallace B., Asymmetric Enforcement of Cooperation in a Social Dilemma forthcoming in: Southern Economic Review, In Press.

2009/19: Magen S., Rechtliche und ökonomische Rationalität im Emissionshandelsrecht

2009/18: Broadberry S.N., Burhop C., Real Wages and Labour Productivity in Britain and Germany, 1871-1938: A Unified Approach to the International Comparison of Living Standards

2009/17: Glöckner A., Hodges S.D., Parallel Constraint Satisfaction in Memory-Based Decisions

2009/16: Petersen N., Review Essay: How Rational is International Law?

forthcoming in: European Journal of International Law, vol. 20, In Press.

2009/15: Bierbrauer F., On the legitimacy of coercion for the financing of public goods

2009/14: Feri F., Irlenbusch B., Sutter M., Efficiency Gains from Team-Based Coordination - Large-Scale Experimental Evidence

2009/13: Jansen J., On Competition and the Strategic Management of Intellectual Property in Oligopoly

2009/12: Hellwig M., Utilitarian Mechanism Design for an Excludable Public Good published in: Economic Theory, vol. 2009, no. July 14, Berlin/Heidelberg, Springer, 2009.

2009/11: Weinschenk P., Persistence of Monopoly and Research Specialization

2009/10: Horstmann N., Ahlgrimm A., Glöckner A., How Distinct are Intuition and Deliberation? An Eye-Tracking Analysis of Instruction-Induced Decision Modes

2009/09: Lübbers T., Is Cartelisation Profitable? A Case Study of the Rhenish Westphalian Coal Syndicate, 1893-1913

2009/08: Glöckner A., Irlenbusch B., Kube S., Nicklisch A., Normann H., Leading with(out) Sacrifice?

A Public-Goods Experiment with a Super-Additive Player

forthcoming in: Economic Inquiry, In Press.

2009/07: von Weizsäcker C., Asymmetrie der Märkte und Wettbewerbsfreiheit

2009/06: Jansen J., Strategic Information Disclosure and Competition for an Imperfectly Protected Innovation forthcoming in: Journal of Industrial Economics, In Press. 
2009/05: Petersen N., Abkehr von der internationalen Gemeinschaft? - Die aktuelle Rechtsprechung des US Supreme Court zur innerstaatlichen Wirkung von völkerrechtlichen Verträgen -

forthcoming in: Völkerrecht im innerstaatlichen Bereich, Vienna, facultas.wuv, In Press.

2009/04: Rincke J., Traxler C., Deterrence Through Word of Mouth

2009/03: Traxler C., Winter J., Survey Evidence on Conditional Norm Enforcement

2009/02: Herbig B., Glöckner A., Experts and Decision Making: First Steps Towards a Unifying Theory of Decision Making in Novices, Intermediates and Experts

2009/01: Beckenkamp M., Environmental dilemmas revisited: structural consequences from the angle of institutional ergonomics, issue 2009/01

\section{Preprints 2008}

2008/49: Glöckner A., Dickert S., Base-rate Respect by Intuition: Approximating Rational Choices in Base-rate Tasks with Multiple Cues

2008/48: Glöckner A., Moritz S., A Fine-grained Analysis of the Jumping to Conclusions Bias in Schizophrenia: DataGathering, Response Confidence, and Information Integration

2008/47: Hellwig M., A Generalization of the Atkinson-Stiglitz (1976) Theorem on the Undesirability of Nonuniform Excise Taxation

2008/46: Burhop C., The Underpricing of Initial Public Offerings in Imperial Germany, 1870-1896

forthcoming in: German Economic Review, In Press.

2008/45: Hellwig M., A Note on Deaton's Theorem on the Undesirability of Nonuniform Excise Taxation forthcoming in: Economics Letters, In Press.

2008/44: Hellwig M., Zur Problematik staatlicher Beschränkungen der Beteiligung und der Einflussnahme von Investoren bei großen Unternehmen

published in: Zeitschrift für das gesamte Handelsrecht und Wirtschaftsrecht, vol. 172, pp. 768-787, 2008.

2008/43: Hellwig M., Systemic Risk in the Financial Sector: An Analysis of the Subprime-Mortgage Financial Crisis published in: De Economist, no. 16.07.2009: Springer US, 2009.

published in: Jelle Zijlstra Lecture, no. 2008/5, Wassenaar, NL, Netherlands Institute for Advanced Study in the Humanities and Social Sciences, Institute of the Royal Netherlands Academy of Arts and Sciences, pp. 100, 2008.

2008/42: Glöckner A., Herbold A., Information Processing in Decisions under Risk: Evidence for Compensatory Strategies based on Automatic Processes

2008/41: Lüdemann J., Magen S., Effizienz statt Gerechtigkeit forthcoming in: Zeitschrift für Rechtsphilosophie, In Press.

2008/40: Engel C., Die Bedeutung der Verhaltensökonomie für das Kartellrecht

2008/39: Bierbrauer F., A unified approach to the revelation of public goods preferences and to optimal income taxation

2008/38: Burhop C., Lübbers T., Incentives and Innovation? R\&D Management in Germany’s High-Tech Industries During the Second Industrial Revolution

forthcoming in: Explorations in Economic History, In Press.

2008/37: Nicklisch A., Salz T., Reciprocity and status in a virtual field experiment

2008/36: Glöckner A., Engel C., Can We Trust Intuitive Jurors? An Experimental Analysis 